This item was submitted to Loughborough's Research Repository by the author.

Items in Figshare are protected by copyright, with all rights reserved, unless otherwise indicated.

\title{
Turbulence at the Lee bound: maximally non-normal vortex filaments and the decay of a local dissipation rate
}

PLEASE CITE THE PUBLISHED VERSION

https://doi.org/10.1017/jfm.2019.779

\section{PUBLISHER}

Cambridge University Press

VERSION

AM (Accepted Manuscript)

\section{PUBLISHER STATEMENT}

This paper was accepted for publication in the journal Journal of Fluid Mechanics and the definitive published version is available at https://doi.org/10.1017/jfm.2019.779

LICENCE

CC BY-NC-ND 4.0

\section{REPOSITORY RECORD}

Keylock, Chris. 2019. "Turbulence at the Lee Bound: Maximally Non-normal Vortex Filaments and the Decay of a Local Dissipation Rate”. figshare. https://hdl.handle.net/2134/11871921.v1. 


\title{
Turbulence at the Lee bound: Maximally non-normal vortex filaments and the decay of a local dissipation rate
}

\author{
Christopher J. Keylock
}

February 19, 2020

\begin{abstract}
This paper is concerned with the properties of vortical regions in homogeneous, isotropic turbulence, defined as the locations where the eigenvalues of the velocity gradient tensor (VGT) include a conjugate pair. This means that there is a closed streamline in the Lagrangian frame. A tight mathematical bound on the degree of non-normality of the VGT is used to classify flow according to whether or not this bound is attained $(\ell=1)$ or not $(\ell=0)$. The $\ell=1$ condition is attained trivially when the eigenvalues of the VGT are all real because of an absence of a normal component to the vorticity. In the vortical regions, this condition arises in a transient and spatially limiited manner if total strain exceeds enstrophy ( $Q$ is negative). However, when enstrophy exceeds total strain ( $\mathrm{Q}$ is positive) and particularly where there is a positive balance between strain production and enstrophy production ( $R$ is negative), the $\ell=1$ state is obtained more frequently and in a spatially coherent manner that generates rod-like filaments within vortical tubes. An analysis of the dynamically relevant terms using a recently developed formulation [Keylock, C.J. (2018). The Schur decomposition of the velocity gradient tensor for turbulent flows, J. Fluid Mech. 848, 876-904], highlights the controls on these dynamics. The bound is attained when normal total strain decreases rather than non-normality increases. Because turbulent dissipation is related to fluid straining, the near-absence of normal total strain means any observed strain is a consequence of the non-local contribution to the tensorial dynamics. Thus, there is a source of intermittency in the dynamics of dissipation that is hidden in standard analyses. It is shown that of the two terms that contribute to the non-normal production dynamics, it is the one that is typically smallest in magnitude that is of greatest importance within these filaments. The centroid of these filaments lies, on average, a quarter of the radius of the enclosing flow structure away from its own centroid, with the typical distance between filament centroids scaling at just under a Taylor scale. This latter result contributes to an understanding of how the structuring of flow topology at the Taylor scale helps explain smaller scale dissipation.
\end{abstract}




\section{Introduction}

In 1935, Geoffrey Taylor outlined his statistical theory for turbulence, which included the definition of what became known as the Taylor length scale, $\lambda$. He stated that this length corresponds to the diameter of the smallest eddies responsible for the dissipation of energy [Taylor, 1935]. Subsequent work established that the characteristic length scale for the smallest eddies is the Kolmogorov scale, $\eta=\left(\nu^{3} / \epsilon\right)^{1 / 4}$, where $\nu$ is the kinematic viscosity and $\epsilon$ is the dissipation rate [Kolmogorov, 1941]. However, there has been increasing interest in the

role of the Taylor scale for turbulence dissipation in recent years in terms of spectral-based studies of energy transfer [George, 1992], the discovery that the Einstein-Markov coherence length for turbulence is typically $\sim 0.8 \lambda$ [Lück et al., 2006], and the result that the maximum dissipation length is similar to the Taylor scale and exhibits a square-root scaling with Reynolds number [Yakhot, 2003]. In addition, there is work showing that the dissipation rate coefficient can also be related to the stagnation point structure in the flow, which in turn implies a Taylor length scaling because the mean separation of stagnation points is proportional to $\lambda$ [Goto and Vassilicos, 2009]. Hence, the fluctuating velocity is much rougher at scales larger than $\lambda$ than at smaller scales, providing the rationale for intermittency-based studies of turbulence [Frisch et al., 1978, Meneveau and Sreenivasan, 1987, Lashermes et al., 2008]. The link that Goto and Vassilicos [2009] established between dissipation coefficient and stagnation structure is a statement about flow topology because the large eddies in the flow determine this structure. Research undertaken by Elsinga and Marusic [2010] looked into the small-scale properties of turbulent boundary layers, channel flows and homogeneous, isotropic turbulence (HIT), using simulations and particle imaging velocimetry (PIV) data. Their results supported a model for turbulence where vortices are found at the edge of uniform flow regions, and for all flows studied, the distance between vortex cores was between $1.4 \lambda$ and $1.8 \lambda$, consistent with the imprint of the large eddies on the stagnation point structure.

It is not immediately clear from the basic equations why such structures play a role in dissipation because local dissipation is defined as

$$
\epsilon=\nu \operatorname{tr}\left(S_{A}^{2}\right),
$$

where the strain rate is

$$
\mathrm{S}_{A}=\frac{1}{2}\left(\mathrm{~A}+\mathrm{A}^{*}\right),
$$

with the velocity gradient tensor (VGT), A, is given by $A_{i j}=\partial u_{i} / \partial x_{j}$, where $u$ is an orthogonal velocity component, $x$ is an orthogonal spatial direction, and the asterisk is the transpose. To complete this picture, the additive decomposition of the VGT into Hermitian (strain rate) and skew-Hermitian (rotation rate) components is

$$
\mathrm{A}=\mathrm{S}_{A}+\Omega_{A},
$$


where

$$
\Omega_{A}=\frac{1}{2}\left(\mathrm{~A}-\mathrm{A}^{*}\right) .
$$

While (1) shows that rotation rate, vorticity or enstrophy plays no direct role in local dissipation, the equation for the evolution of the total strain contains terms corresponding to both strain production, $-\operatorname{det}\left(\mathrm{S}_{A}\right)$, and enstrophy production, $\operatorname{tr}\left(\Omega_{A}^{2} \mathrm{~S}_{A}\right)$, [Tsinober, 2001]

$$
\frac{1}{2} \frac{\mathrm{D} \operatorname{tr}\left(\mathrm{S}_{A}^{2}\right)}{\mathrm{D} t}=-3 \operatorname{det}\left(S_{A}\right)-\frac{1}{4} \operatorname{tr}\left(\Omega_{A}^{2} \mathrm{~S}_{A}\right)-\mathrm{S}_{A} \frac{\partial^{2} p}{\partial x_{i} \partial x_{j}}+\nu \mathrm{S}_{A} \nabla^{2} \mathrm{~S}_{A},
$$

and there is a long history to considering the role of enstrophy production in dissipation physics [Taylor, 1938b]. In particular, the relative alignment between the vortical and straining components of the flow are a crucial for determining the magnitude of this term [Hamlington et al., 2008]. A key result from the early numerical investigations into the properties of homogeneous, isotropic turbulence (HIT) was the preferential alignment between the vorticity vector and the strain eigenvector corresponding to the intermediate strain eigenvalue [Kerr, 1985, Ashurst et al., 1987]. Ohkitani [2002] has shown the importance of viscosity for preserving this alignment between the vorticity vector and the intermediate eigenvector, highlighting the limitations of models based on the Euler equations for fully capturing the dynamics of turbulence. While the evolution of total strain is (indirectly) dependent on enstrophy production and thus, the behaviour of vortices in the flow, the evolution of the enstrophy itself is directly proportional to the enstrophy production

$$
\frac{1}{2} \frac{\mathrm{D} \operatorname{tr}\left(\Omega_{A}^{2}\right)}{\mathrm{D} t}=\operatorname{tr}\left(\Omega_{A}^{2} \mathrm{~S}_{A}\right)+\nu \Omega_{A} \nabla^{2} \Omega_{A},
$$

and the mean enstrophy production, $\left\langle\operatorname{tr}\left(\Omega_{A}^{2} \mathrm{~S}_{A}\right)\right\rangle$ is positive [Taylor, 1938b].

This paper is concerned with the role of vortical motions in dissipation physics, but is focussed on the partitioning of the total strain and enstrophy at a point in the flow field between local and non-local processes. Nonlocality is, with nonlinearity and non-integrability, one of the "three Ns" of turbulence [Tsinober, 2009] that make the analysis of the Navier-Stokes equations so difficult. The novel aspect of our approach is to introduce a mathematical bound on the behaviour of the velocity gradient tensor (VGT). We then examine the manner in which the bound is attained, and the typical statistics for the flow when the bound is attained compared to other times and locations. We show that the Taylor scale plays a role in determining the maximum size of the regions that attain the bound and, how the genuinely local contribution to strain virtually disappears in such regions, while the values for total strain and the production terms, $\operatorname{tr}\left(\Omega_{A}^{2} \mathrm{~S}_{A}\right)$ and $-\operatorname{det}\left(\mathrm{S}_{A}\right)$, remain essentially constant. In other words, there is an intermittency to dissipation, not in terms of the standard conceptualization of this phenomenon [Kolmogorov, 1962, Frisch et al., 1978], but in terms of the partitioning of the total strain between local and nonlocal contributions and the decline of the former. 


\section{The Lee bound for the velocity gradient ten- sor}

There are typically two approaches to the analysis of turbulent flows in terms of the VGT. The first focusses on the decomposition into strain rate and rotation rate tensors as discussed above, where it is also common to express the rotation tensor as a vorticity vector, $\boldsymbol{\omega}$, where each term is

$$
\omega_{i}^{(A)}=-\epsilon_{i j k} \Omega_{j k}^{(A)},
$$

and $\epsilon_{i j k}$ is the Levi-Cevita symbol. The second approach considers the invariants of the characteristic equation of $\mathrm{A}$

$$
e_{i}^{3}+\mathrm{P}_{A} e_{i}^{2}+\mathrm{Q}_{A} e_{i}+\mathrm{R}_{A}=0,
$$

which intrinsically requires a consideration of the eigenvalues, $e_{i}$. For incompressible turbulence, $\mathrm{P}_{A}=-\sum e_{i}=0$, leading to a compact expression for the discriminant function separating regions where the eigenvalues are real $(\Delta<0)$ and where they form a conjugate pair $(\Delta>0)$ [Chong et al., 1990, Rabey et al., 2015]:

$$
\Delta=\mathrm{Q}_{A}^{3}+\frac{27}{4} \mathrm{R}_{A}^{2} .
$$

It is then possible to write equations for the Lagrangian evolution of the flow in terms of the remaining two invariants, and their constituent terms [Girimaji and Pope, 1990, Martin et al., 1998, Biferale et al., 2007, Li and Meneveau, 2007, Chevillard et al., 2008, Wilczek and Meneveau, 2014, Johnson and Meneveau, 2016]. The most celebrated approach due to the need to only consider the invariants is the restricted Euler model [Cantwell, 1992]

$$
\begin{aligned}
\frac{d \mathrm{Q}_{A}}{d t} & =-3 \mathrm{R}_{A} \\
\frac{d \mathrm{R}_{A}}{d t} & =\frac{2}{3} \mathrm{Q}_{A}^{2},
\end{aligned}
$$

where it is assumed that viscous effects may be ignored and that the pressure Hessian is isotropic. This model replicates a number of the features of turbulence [Meneveau, 2011], but cannot capture the observed clock-wise circulation of Lagrangian trajectories around a space formed from $\mathrm{Q}_{A}$ and $\mathrm{R}_{A}$, which is demonstrated later in this paper. This is because trajectories maintain a constant value for $\Delta$ in the restricted Euler model. In addition, the model is unable

to capture the role of viscous effects [Ohkitani, 2002] or the deviatoric part of the pressure Hessian on the preservation of the alignment between the vorticity vector and the intermediate strain eigenvector.

It has recently been suggested that there are advantages in introducing an additive decomposition of the velocity gradient tensor, $\mathrm{A}=\mathrm{B}+\mathrm{C}$ that isolates the eigenvalue-related, normal contributions in $B$, with $C$ containing the nonnormal contributions [Keylock, 2018]. This approach is based on a complex 
Schur decomposition of A [Schur, 1909], given by

$$
\mathrm{A}=\mathrm{UTU}^{*} \text {, }
$$

where $\mathrm{U}$ is unitary and the upper triangular tensor $\mathrm{T}=\mathrm{E}+\mathrm{N}$ consists of a diagonal matrix of eigenvalues, $\mathrm{E}$, where $E_{i, i}=e_{i}$, and the non-normal contribution, $\mathrm{N}$. Decomposing $\mathrm{T}$ in this way leads to

$$
\begin{aligned}
& B=U_{E U^{*}} \\
& C=U_{N U^{*}} .
\end{aligned}
$$

This permits, for example, a simple rewriting of the restricted Euler model as

$$
\begin{aligned}
& \frac{d \mathrm{Q}_{B}}{d t}=-3 \mathrm{R}_{B} \\
& \frac{d \mathrm{R}_{B}}{d t}=\frac{2}{3} \mathrm{Q}_{B}^{2} .
\end{aligned}
$$

That the non-normal contributions from $\mathrm{C}$ are excluded from the restricted Euler model highlights that the effects captured in the non-normal term are those due to viscosity and the deviatoric part of the pressure Hessian, i.e. the non-local aspects of the turbulence structure [Ohkitani and Kishiba, 1995, Hamlington et al., 2008].

\subsection{Non-normality and the Lee bound}

Taking the Frobenius norm of $\mathrm{N}$, i.e. $n=\|\mathrm{N}\| \equiv \sqrt{\operatorname{tr}\left(\mathrm{NN}^{*}\right)}$, leads to one form for tensor non-normality. An alternative is given by

$$
h=\left\|\mathrm{AA}^{*}-\mathrm{A}^{*} \mathrm{~A}\right\|,
$$

and there has been a significant amount of work formulating bounds for $n$ given $h$ or vice versa [Henrici, 1962, Eberlein, 1965, Kress et al., 1974]. A tight bound was proposed by Lee [1995], which considers complex shifts of A and also uses the Hermitian/skew-Hermitian decomposition of the tensor. The latter is important because non-normality is partitioned equally between strain and rotation components in this frame [Keylock, 2018, Dong et al., 2019], leading to

$$
\begin{aligned}
\left\|S_{A}\right\|^{2} & =\|\operatorname{Re}\{\mathrm{E}\}\|^{2}+\frac{1}{2}\|\mathrm{~N}\|^{2} \\
\left\|\Omega_{A}\right\|^{2} & =\|\operatorname{Im}\{\mathrm{E}\}\|^{2}+\frac{1}{2}\|\mathrm{~N}\|^{2},
\end{aligned}
$$

where Re and Im are the real and imaginary parts.

Introducing a complex scalar, $\alpha$, such that the perturbed VGT is $\mathrm{A}-\alpha \mathrm{l}$, where $\mathbf{I}$ is the identity matrix, the perturbed strain and rotation tensors are given, respectively, by $\mathrm{S}_{A}-\operatorname{Re}(\alpha) \mathrm{I}$ and $\Omega_{A}-\operatorname{Im}(\alpha) \mathrm{I}$. The upper Lee bound for $n, b^{L}(n)$, is

$$
b^{L}(n)=b^{L}(\mathrm{~A}-\alpha \mathrm{l})=\min \left\{\begin{array}{l}
\sqrt{2}\left(\left\|\mathrm{~S}_{A}-\operatorname{Re}\{\alpha\} \mid\right\|^{2}-\|\operatorname{Re}\{\mathrm{E}\}-\operatorname{Re}\{\alpha\}\| \|^{2}\right)^{\frac{1}{2}} \\
\sqrt{2}\left(\left\|\Omega_{A}-i \operatorname{Im}\{\alpha\}\right\|\left\|^{2}-\right\| \operatorname{Im}\{\mathrm{E}\}-\operatorname{Im}\{\alpha\} \|^{2}\right)^{\frac{1}{2}}
\end{array} .\right.
$$


Taking each of these expressions and minimizing the term containing the eigenvalues establishes that

$$
\alpha=\frac{1}{3} \sum \operatorname{Re}\left\{e_{i}\right\}+\frac{1}{3} i \sum \operatorname{Im}\left\{e_{i}\right\}=\frac{1}{3} \operatorname{tr}(\mathrm{A}) .
$$

For incompressible turbulence, where $\operatorname{tr}(A)=0$, the Lee bound may be simplified to the following, tight expression:

$$
b^{L}(n) \leq \sqrt{2} \min \left(\left\|\mathrm{S}_{A}\right\|,\left\|\Omega_{A}\right\|\right) .
$$

In terms of the invariants in (8), and given that

$$
\mathrm{Q}_{A}=\frac{1}{2}\left(\left\|\Omega_{A}\right\|^{2}-\left\|\mathrm{S}_{A}\right\|^{2}\right)
$$

it follows that $\left\|\mathrm{S}_{A}\right\|$ will control the bound in rotational regions with positive $\mathrm{Q}_{A}$, and $\left\|\Omega_{A}\right\|$ dictates the bound for negative $\mathrm{Q}_{A}$. Beneath the discriminant function, where $\Delta<0$, it can be shown that $\Omega_{B}$, does not exist [Keylock, 2018]. Given the equipartition of non-normality between strain and rotation in (17) it then follows from (20) that for $\Delta<0, b^{L}=\sqrt{2}\left\|\Omega_{C}\right\|=\sqrt{2}\left\|\mathrm{~S}_{C}\right\|$. Hence, the Lee bound must be fulfilled in this region. However, where $\Delta>0$ no such constraint exists and the attainment of the bound in these vortical regions is non-trivial. This forms the focus of this paper.

\subsection{Vortical flow regions and attainment of the Lee bound}

In this paper, for consistency with the above statement, we adopt a common, practical definition of a vortical region in the flow as one where a conjugate pair of eigenvalues exists, i.e. $\Delta>0$ [Chong et al., 1990]. This means that in the Lagrangian frame, there is a closed streamline. Such a definition also underpins other measures of coherent structures that are based on the imaginary part of the eigenvalues [Zhou et al., 1999, Chakraborty et al., 2005]. Thus, given $\Delta>0$, we can define the degree to which the Lee bound is attained as

$$
\Phi=\frac{\|\mathrm{N}\|}{\sqrt{2} \min \left(\left\|\mathrm{S}_{A}\right\|,\left\|\Omega_{A}\right\|\right)},
$$

on the understanding that where $\mathrm{Q}_{A}>0$ the bound is imposed by $\left\|\mathrm{S}_{A}\right\|$ and where $\mathrm{Q}_{A}<0$ it is based on $\left\|\Omega_{A}\right\|(21)$, with the bound obtained in a trivial fashion for $\Delta<0$. From this, we consider the practical attainment of the Lee bound to be indicated by the binary variable, $\ell$, where

$$
\begin{aligned}
& \ell=1 \text { if } \Phi>0.9999 \\
& \ell=0 \text { if } \Phi \leq 0.9999 .
\end{aligned}
$$


Table 1: Summary properties of the HIT simulation in the Johns Hopkins database [Li et al., 2008]. All quantities are dimensionless.

\begin{tabular}{lc}
\hline Property & Value \\
\hline Grid & $1024^{3}$ periodic box \\
Domain & {$[0,2 \pi]^{3}$} \\
Viscosity, $\nu$ & $1.85 \times 10^{-4}$ \\
Mean dissipation rate, $\epsilon$ & 0.0928 \\
Taylor micro-scale, $\lambda$ & 0.114 \\
Taylor Reynolds number, $R e_{\lambda}$ & 433 \\
Kolmogorov length, $\eta$ & $2.87 \times 10^{-3}$ \\
\hline
\end{tabular}

\section{$3 \quad$ Numerical Simulation and Eulerian Results}

\subsection{Direct Numerical Simulation}

This study makes use of the Johns Hopkins Turbulence Database direct numerical simulation of steady homogeneous, isotropic turbulence (HIT) at a Taylor Reynolds number of 433 [Li et al., 2008, Wan et al., 2016], which has been employed in a number of other turbulence studies [Lüthi et al., 2009, Wan et al., 2010, Keylock, 2017, Ballouz and Ouellette, 2018]. The simulation was performed on a $1024^{3}$ grid using a pseudo-spectral method. The energy was injected to maintain the total energy in the Fourier modes, while also retaining a wave number magnitude less than or equal to 2 in each mode. Some properties of the simulation are summarized in Table 1.

\subsection{Attainment of the Lee bound in $\mathrm{Q}_{A}-\mathbf{R}_{A}$ space}

To illustrate how efficient different regions in $\mathrm{Q}_{A}-\mathrm{R}_{A}$ space are at attaining the Lee bound, Fig. 1 is a sketch of the $\mathrm{Q}_{A}-\mathrm{R}_{A}$ diagram, with regions identified according to the typology adopted in our previous work [Keylock, 2018]:

- Region $1-\mathrm{Q}_{A}>0, \mathrm{R}_{A}>0$;

- Region $2-\mathrm{Q}_{A}>0, \mathrm{R}_{A}<0$;

- Region $3-\mathrm{Q}_{A}<0, \Delta>0, \mathrm{R}_{A}<0$;

- Region $4-\mathrm{Q}_{A}<0, \Delta<0, \mathrm{R}_{A}<0$;

- Region $5-\mathrm{Q}_{A}<0, \Delta>0, \mathrm{R}_{A}>0$;

- Region $6-\mathrm{Q}_{A}<0, \Delta<0, \mathrm{R}_{A}>0$.

The dashed line in Fig. 1 is the discriminant, $\Delta=0$, and beneath it, corresponding to $\sim 40 \%$ of VGTs, $\ell=1$ is imposed by definition as discussed above. However, for $\Delta>0$ the average occurrence over all regions is $\sim 0.7 \%$ of tensors, 


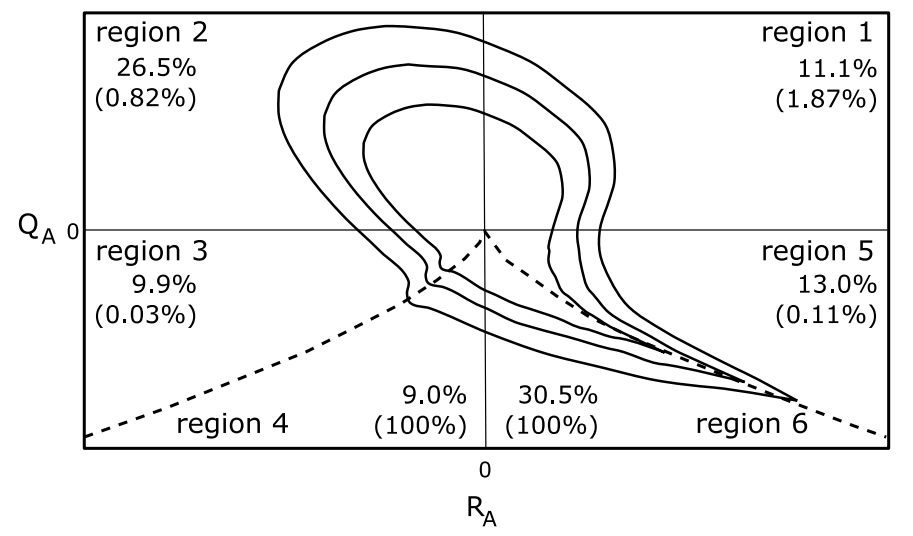

Figure 1: A sketch of the $\mathrm{Q}_{A}-\mathrm{R}_{A}$ diagram showing contours for the typical joint distribution function, as well as the percentage occupancy of each region for the HIT numerical simulation and the efficiency of attaining the Lee bound (values in brackets). Thus, $1.87 \%$ of occurrences in region 1 attain $\ell=1$. The dashed line indicates the discriminant, $\Delta=0$, which defines the divide between regions 3 and 4 , and 5 and 6 , respectively. The Vieillefosse tail is depicted by the contours extending along the discriminant between regions 5 and 6 .

facilitated by $\mathrm{R}_{A}>0$ and $\mathrm{Q}_{A}>0$. Thus, the net effect of the more efficient generation of $\ell=1$ states in region 1 is that regions 1 and 2 generate occurrences at the Lee bound at the same frequency as each other despite the 11.1 to 26.5 difference in relative frequency. In effect, this acts to induce a symmetry to the $\mathrm{Q}_{A}-\mathrm{R}_{A}$ diagram in the enstrophy-dominated regions if only the $\ell=1$ occurrences are considered. This potentially implies a relation between the $\ell=1$ occurrences and energetic flow structures as work using a triple decomposition into mean, coherent, and fluctuating velocities, and extracting the coherent part using proper orthogonal decomposition, has found that the $\mathrm{Q}_{A}-\mathrm{R}_{A}$ diagram is approximately symmetric for the coherent part as shown in Fig. 6 of Buxton et al. [2017]. We examine the relation between $\ell=1$ occurrences and coherent flow structures in greater detail below.

The third invariant of the characteristic equation for the velocity gradient tensor, $\mathrm{R}_{A}$, may be decomposed into its constituent strain production and enstrophy production terms, i.e.

$$
\mathrm{R}_{A}=-\operatorname{det}\left(\mathrm{S}_{A}\right)-\operatorname{tr}\left(\Omega_{A}^{2} \mathrm{~S}_{A}\right),
$$

and Table 2 sub-divides the results for each region of the $\mathrm{Q}_{A}-\mathrm{R}_{A}$ diagram by the signs for these production terms, while also highlighting the differences that arise when $\ell=1$. The region that is in strongest agreement with the results of Taylor [1938a] and Betchov [1956] for the mean values is region 5, where 
Table 2: Proportional occupany of different regions of the $\mathrm{Q}_{A}-\mathrm{R}_{A}$ diagram as a function of region as well as strain production state and enstrophy production state. The sum of the values in each row of columns four to seven is 1.0. The second column therefore provides a means to weight the values in those columns to determine the proportional occupancy for all VGTs in HIT. The one value italicized in each row is the one possibility for the signs of $-\operatorname{det}\left(\mathrm{S}_{B}\right)$ and $\operatorname{tr}\left(\Omega_{B}^{2} S_{B}\right)$ for each region, Overall results are in the upper row for each region, and the results for $\ell=1$ are given beneath this.

\begin{tabular}{ccccccc}
\hline Region & $\begin{array}{c}\text { \% of all } \\
\text { VGTs }\end{array}$ & $\begin{array}{c}\mathrm{R}_{A}^{(S)}>0 \\
\operatorname{tr}\left(\Omega_{A}^{2} \mathrm{~S}_{A}\right)>0\end{array}$ & $\begin{array}{c}\mathrm{R}_{A}^{(S)}>0 \\
\operatorname{tr}\left(\Omega_{A}^{2} \mathrm{~S}_{A}\right)<0\end{array}$ & $\begin{array}{c}\mathrm{R}_{A}^{(S)}<0 \\
\operatorname{tr}\left(\Omega_{A}^{2} \mathrm{~S}_{A}\right)<0\end{array}$ & $\begin{array}{c}\mathrm{R}_{A}^{(S)}<0 \\
\operatorname{tr}\left(\Omega_{A}^{2} S_{A}\right)>0\end{array}$ \\
\hline 1 & 11.1 & all & 0.16 & 0.37 & 0.47 & - \\
& & $\ell=1$ & 0.52 & 0.06 & 0.42 & - \\
2 & 26.5 & all & 0.75 & - & 0.03 & 0.22 \\
3 & & $\ell=1$ & 0.63 & - & 0.32 & 0.05 \\
& \multirow{2}{*}{5.9} & all & 0.54 & - & 0.10 & 0.36 \\
& \multirow{2}{*}{13.0} & $\ell=1$ & 0.67 & - & 0.24 & 0.09 \\
& & all & 0.54 & 0.38 & 0.08 & - \\
\hline
\end{tabular}

$94 \%$ of tensors had positive values for both strain production and enstrophy production. A similar magnitude of change is also seen for region 1, and the even clearer result is the near absence of cases where these terms are opposite in sign once $\ell=1$.

To investigate this further, it is useful to expand the terms constituting $\mathrm{Q}_{A}$ and $\mathrm{R}_{A}$ to isolate the contributions from normal and non-normal terms. The second invariant of the VGT is traditionally written as stated in (21). Following Keylock [2018] we may instead state that

$$
\mathrm{Q}_{A}=\frac{1}{2}\left[\left(\left\|\Omega_{B}\right\|^{2}+\left\|\Omega_{C}\right\|^{2}\right)-\left(\left\|\mathrm{S}_{B}\right\|^{2}+\left\|\Omega_{C}\right\|^{2}\right)\right],
$$

i.e. $\mathrm{Q}_{A}=\mathrm{Q}_{B}$, where

$$
\mathrm{Q}_{B}=\frac{1}{2}\left(\left\|\Omega_{B}\right\|^{2}-\left\|\mathrm{S}_{B}\right\|^{2}\right)
$$

and the non-normal contribution cancels in the restricted Euler analysis. With regard to the production terms, we expand (24) as

$$
\begin{aligned}
& -\operatorname{det}\left(\mathrm{S}_{A}\right)=-\operatorname{det}\left(\mathrm{S}_{B}\right)-\operatorname{det}\left(\mathrm{S}_{C}\right)+\operatorname{tr}\left(\Omega_{C}^{2} \mathrm{~S}_{B}\right) \\
& \operatorname{tr}\left(\Omega_{A}^{2} \mathrm{~S}_{A}\right)=\operatorname{tr}\left(\Omega_{B}^{2} \mathrm{~S}_{B}\right)-\operatorname{det}\left(\mathrm{S}_{C}\right)+\operatorname{tr}\left(\Omega_{C}^{2} \mathrm{~S}_{B}\right) .
\end{aligned}
$$

The first term on the right-hand side of each equation in (27) is the normal term (strain production then enstrophy production), while the second and third 

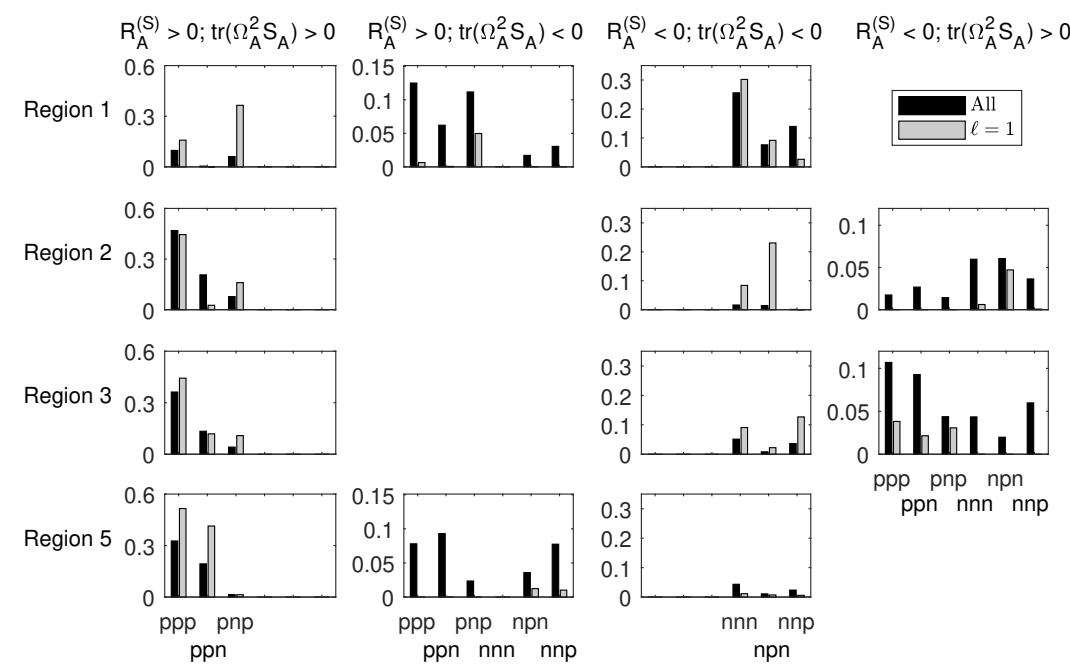

ppn nnn nnp

Figure 2: A partitioning of the results in Table 2 by the values for the interaction production and non-normal production terms, and their sum. The abscissa for each panel indicates the possible sign combinations for these terms ("p" = positive, "n" = negative). From left to right, each block of three characters gives the sign for $\operatorname{tr}\left(\Omega_{C}^{2} S_{B}\right)-\operatorname{det}\left(S_{C}\right), \operatorname{tr}\left(\Omega_{C}^{2} S_{B}\right)$, and $-\operatorname{det}\left(S_{C}\right)$, respectively. The black bars are the results for all the data, while the light grey are the results for $\ell=1$.

terms have been termed the non-normal production and interaction production, respectively [Keylock, 2018]. The signs for the two normal terms in (27) are dictated by the sign for $\mathrm{R}_{A}$ because $\mathrm{R}_{A}=-\operatorname{det}(\mathrm{A})=-\prod e_{i}$, i.e.:

$$
\begin{aligned}
\operatorname{sgn}\left[-\operatorname{det}\left(S_{B}\right)\right] & =\operatorname{sgn}\left(R_{A}\right) \\
\operatorname{sgn}\left[\operatorname{tr}\left(\Omega_{B}^{2} S_{B}\right)\right] & =-\operatorname{sgn}\left(R_{A}\right) .
\end{aligned}
$$

The appropriate combination of these terms is italicized in each row of Table 2 and it is clear that for HIT, only between $22 \%$ and $38 \%$ of cases retain the sign combination for A that is anticipated from the consideration of B. This highlights the importance of the non-normal and interaction terms from (27) for dictating the behaviour of A. For regions 1, 2, and 3, the most frequently occupied category has the same sign for enstrophy production, but a change in the sign for strain production relative to the italicized term. In contrast, for region 5 , it is enstrophy production that changes sign. This is consistent with high strain production close to the Vieillefosse tail [Vieillefosse, 1984], which exists along $\Delta=0$ for $\mathrm{R}_{A}>0$, i.e. between regions 5 and 6 in Fig. 1.

When examining the results for the $\ell=1$ regions in Table 2 , the effect of nonnormality is very marked with only between $3 \%$ and $9 \%$ of cases retaining the 
sign combination anticipated based on the signs for $-\operatorname{det}\left(\mathrm{S}_{B}\right)$ and $\operatorname{tr}\left(\Omega_{B}^{2} \mathrm{~S}_{B}\right)$. While one would expect a large effect of the non-normal contributions on the dynamics for the $\ell=1$ regions by definition, it is not necessarily the case that this would change the signs of $-\operatorname{det}\left(\mathrm{S}_{A}\right)$ and $\operatorname{tr}\left(\Omega_{A}^{2} \mathrm{~S}_{A}\right)$ because the non-normal and interaction production terms could act in a similar direction to the normal production terms. Instead, we find that between $52 \%$ and $94 \%$ of the VGTs in a given region with $\ell=1$ have positive values for both $-\operatorname{det}\left(\mathrm{S}_{A}\right)$ and $\operatorname{tr}\left(\Omega_{A}^{2} \mathrm{~S}_{A}\right)$, with between $91 \%$ and $97 \%$ of cases having the same sign for these two production terms. This implies a pattern to the mutual alignment structure of the respective vorticity vectors and strain eigenvectors, which is examined further below.

To understand in more detail how the terms in (27) lead to this result, we disaggregate each grouping from Table 2 into the signs ("p" for positive, " $\mathrm{n}$ " for negative) of the interaction production and non-normal production terms, and their sum in Fig. 2, where the black bars are the results for all VGTs and the light grey are for the $\ell=1$ cases. The terms are ordered such that the label "pnp" on the abscissa means that the sum of interaction production and nonnormal production is positive (first "p"), interaction production is negative, and non-normal production is positive (final "p"). The signs of the normal terms for each region are given in (28). Hence, in region 1, where normal strain production is already positive, the non-normal production and interaction production terms must counteract the sign for $\operatorname{tr}\left(\Omega_{B}^{2} S_{B}\right)$ in order to give a positive $\operatorname{tr}\left(\Omega_{A}^{2} S_{A}\right)$. It is clear from the top-left panel of Fig. 2 that relative to all VGTs in region 1 , the $\ell=1$ cases are particularly prone to have a negative interaction production and positive non-normal production, with the latter so great that not only is the sum of these two terms positive but $\operatorname{tr}\left(\Omega_{A}^{2} \mathrm{~S}_{A}\right)$ is also positive. This has arisen at the expense of the probability of both constituents and their sum being positive, with $-\operatorname{det}\left(\mathrm{S}_{A}\right)>0 \operatorname{tr}\left(\Omega_{A}^{2} \mathrm{~S}_{A}\right)<0$.

In region 2 , there is a tendency for both $\operatorname{tr}\left(\Omega_{A}^{2} S_{A}\right)$ and $-\operatorname{det}\left(S_{A}\right)$ to be positive for all cases, but this is focussed to a greater extent on the "ppp" case, although there is still a relative excess for $\ell=1$ states in the "pnp" category for $-\operatorname{det}\left(S_{A}\right)>0$ and $\operatorname{tr}\left(\Omega_{A}^{2} S_{A}\right)>0$ seen in region 1 . However, the mirror image of the result for region 1 also holds: a positive interaction production combines with negative non-normal production to give a negative sum, resulting in $\operatorname{tr}\left(\Omega_{A}^{2} \mathrm{~S}_{A}\right)<0$; this arises largely at the expense of the probability of both constituents and their sum being negative, with $-\operatorname{det}\left(\mathrm{S}_{A}\right)<0$ and $\operatorname{tr}\left(\Omega_{A}^{2} \mathrm{~S}_{A}\right)>0$. In regions 3 and 5 , the dramatic decline in the probability that $-\operatorname{det}\left(\mathrm{S}_{A}\right)$ and $\operatorname{tr}\left(\Omega_{A}^{2} S_{A}\right)$ are opposite in sign is compensated by an increase for $\ell=1$ in the "ppp" case for $-\operatorname{det}\left(S_{A}\right)>0$ and $\operatorname{tr}\left(\Omega_{A}^{2} S_{A}\right)>0$. The difference between these two regions is largely that in region $5, \ell=1$ cases also exhibit a relative excess in the "ppn" state for $-\operatorname{det}\left(S_{A}\right)>0$ and $\operatorname{tr}\left(\Omega_{A}^{2} S_{A}\right)>0$, implying that non-normal production is weak here. The related phenomenon in region 3 is the mirror image case where the "nnp" state for $-\operatorname{det}\left(S_{A}\right)<0$ and $\operatorname{tr}\left(\Omega_{A}^{2} S_{A}\right)<0$ becomes important.

Moving away from an examination of the signs of the constituents, Fig. 3 shows boxplots of the values for the four production terms for regions 1 and 


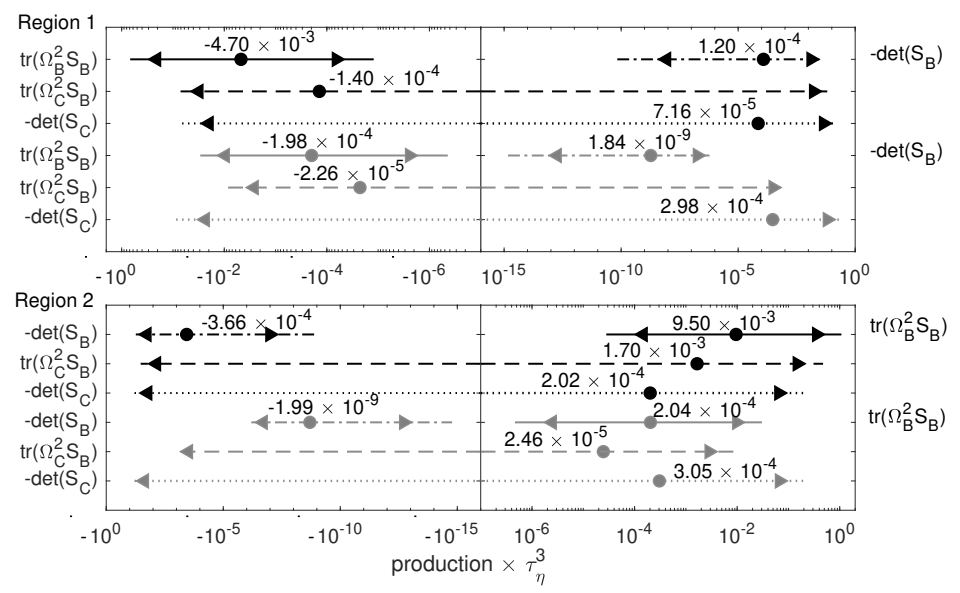

Figure 3: The range of observed values for the normal enstrophy production term (solid lines), normal strain production (dash-dotted lines), interaction production (dashed lines), and non-normal production (dotted lines) in regions 1 and 2. The black lines are for the full dataset and the grey are for the $\ell=1$ cases. All results are non-dimensionalised by $\tau_{\eta}^{3}$, and the lines extend from the 0.5 to the 99.5 percentile with the triangles at the 2.5 and 97.5 percentiles, and the median shown as a circle. The values for the medians are provided above each line. 
2 on logarithmically distributed axes constructed for the negative and positive values separately and then re-combined, with values in grey those that attain the Lee bound. Hence, there is a focus on the two regions that drive the total number of $\ell=1$ instances. Comparing the top rows of the two panels, and noting that the signs of the normal enstrophy production and normal strain production are given by the region, it can be seen that the typical magnitude of the former is, in region 2 , twice that for region 1 , with $\left|\operatorname{det}\left(\mathrm{S}_{B}\right)\right|$ three times as great. Furthermore, interation production in the second row is twelve times greater in magnitude in region 2 , wth a sign difference for the average states, while non-normal production in the third row is 2.8 times greater, but positive on average in both regions. However, it is clear that when the flow attains the $\ell=1$ state (fourth to sixth rows in each panel), these differences in magnitude largely disappear. Values for the normal strain production are tiny, as expected, with those in region 2 only $8 \%$ greater. The equivalent percentage differences for normal enstrophy production, interaction production and non-normal production are $3 \%, 9 \%$, and $2 \%$ respectively. There is also a clear change in the relative magnitude of the production terms in a given region when comparing all cases with the $\ell=1$ states. Normal strain production decreases from third to fourth in magnitude, with non-normal production moving from fourth to first. In this latter case, the median values increase by a factor of 4.2 in region 1 and by $50 \%$ in region 2 . While the median values for interaction production decrease for the $\ell=1$ states compared to all the data, in region 1 this is by a factor of 6 , compared to a factor of 69 in region 2. Similarly, values for normal enstrophy production decrease by a factor of 23.7 in region 1 and 46.6 in region 2 .

The above results suggest that region 1 is innately nearer the $\ell=1$ state compared to region 2 and histograms showing the combined impact of these changes on $\operatorname{tr}\left(\Omega_{C}^{2} S_{B}\right)-\operatorname{det}\left(S_{C}\right)$ are shown in Fig. 4, where the reduced smoothness of the grey lines ( $\ell=1$ cases) is because fewer than $1 \%$ of the overall cases are being sampled. What is clear is that the differences in the combined term for the overall data are a consequence of a typically positive non-normal production in both regions, but an interaction production term with a median that changes sign following that for $\operatorname{tr}\left(\Omega_{B}^{2} S_{B}\right)$, and this declines greatly in magnitude for the $\ell=1$ cases. The effect is particularly marked for the positive values of $\operatorname{tr}\left(\Omega_{C}^{2} S_{B}\right)$, reflecting the aforementioned factor of 6 decline of median interaction production in region 1 but factor of 69 in region 2 . Thus, the explanation for why region 1 is more effective at generating $\ell=1$ states relative to region 2 is that the flow at the Lee bound has a particular and common probability distribution function for $\operatorname{tr}\left(\Omega_{C}^{2} \mathrm{~S}_{B}\right)-\operatorname{det}\left(\mathrm{S}_{C}\right)$ as seen in Fig. 4 and the flow in region 1 is typically nearer this state than that in region 2. This result is useful as it implies that the two terms in (27) with contributions from $\mathrm{C}$ can be treated together for the purpose of understanding the development of $\ell=1$ states as the differences in interaction production seen in Fig. 3 are small enough in magnitude 


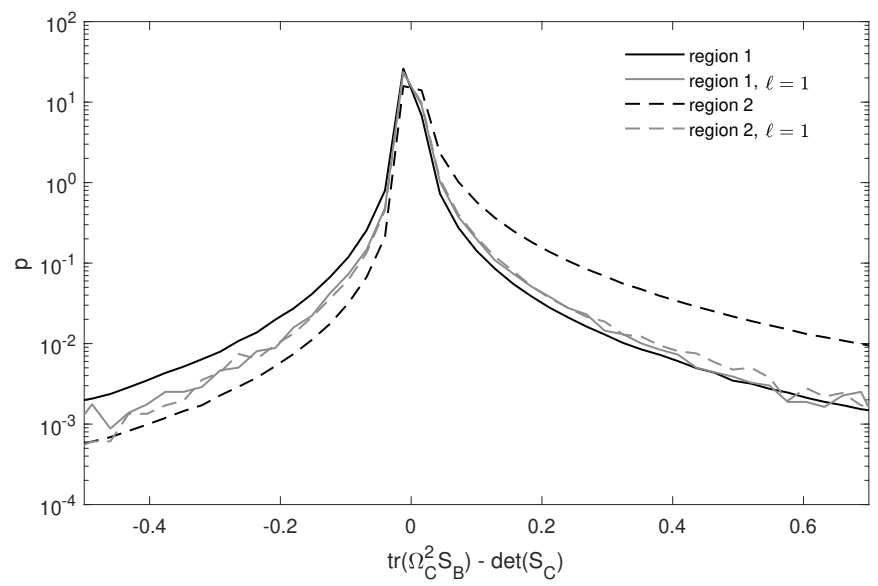

Figure 4: Histograms of the combined effect of the non-normal and interaction production terms in regions 1 (solid lines) and 2 (dashed lines). The black lines are for the full dataset and the grey are for the $\ell=1$ cases. All results are non-dimensionalised by $\tau_{\eta}^{3}$.

\section{Lagrangian Results}

It is informative to undertake a Lagrangian analysis of the trajectories of fluid packets in HIT to determine how, and under what circumstances, the $\ell=1$ states arise. A total of $27^{3}$ fluid packets were tracked for $240 \tau_{\eta}$ at a temporal sampling interval of $0.05 \tau_{\eta}$, with each packet initially spaced $2 \lambda$ from its neighbours. The domain size was $\sim(56 \lambda)^{3}$ so 27 samples could be realised between $\lambda$ and $2 \pi-\lambda$ in each direction.

\subsection{Lagrangian trajectories and durations for the $\ell=1$ states}

Figure 5 shows sixteen Lagrangian trajectory segments, each of which exhibits a change in region in $\mathrm{Q}_{A}-\mathrm{R}_{A}$ space. The arrow shown passes from the region of origin to termination for the chosen trajectory, and the circle half-way along the arrow is the intermediate region where the flow primarily resides while on such a trajectory. All told these sixteen cases accounted for $80.85 \%$ of the total number of trajectories that crossed in to different regions. For each case, the mean time spent in the region denoted by the circle is given in italics and this is in bold if it exceeds $25 \tau_{\eta}$. The well-known, average, clock-wise trajectory through regions of the $\mathrm{Q}_{A}-\mathrm{R}_{A}$ diagram [Lüthi et al., 2009] is discerned visually. It is also the case that when the trajectories are in the upper part of the diagram (regions 1 and 2) they are relatively persistent with mean durations in these two regions ranging 
Table 3: The manner in which the $\ell=1$ state is attained in the positive $\mathrm{Q}_{A}$ part of the $\mathrm{Q}_{A}-\mathrm{R}_{A}$ diagram (corresponding to $91.1 \%$ of cases). The proportions in brackets give the relative frequency of Lagrangian trajectories that attain the $\ell=1$ state for a duration greater than $0.4 \tau_{\eta}$, which is the case for just $0.93 \%$ of cases.

$\begin{array}{cccc}\begin{array}{c}\text { Region immediately } \\ \text { before } \ell=1 \text { occurs }\end{array} & \begin{array}{c}\text { Region at start } \\ \text { of } \ell=1 \text { period }\end{array} & \begin{array}{c}\text { Region at end } \\ \text { of } \ell=1 \text { period }\end{array} & \text { Proportion } \\ 2 & 2 & 1 & 0.316(0.357) \\ 2 & 1 & 1 & 0.170(0.011) \\ 1 & 1 & 2 & 0.139(0.199) \\ 2 & 2 & 2 & 0.089(0.282) \\ 1 & 2 & 2 & 0.082(0.007) \\ 1 & 1 & 1 & 0.076(0.129) \\ 2 & 1 & 2 & 0.020(0.008) \\ 1 & 2 & 1 & 0.019(0.007) \\ & & \text { Total } & 0.910(1.000)\end{array}$

from $28 \leq \tau_{\eta}<58$. This may be contrasted with regions 3 to 6 which account for eleven of the sixteen trajectories and $78 \%$ of the illustrated occurrences, but with a typically reduced duration (an average duration of $\left\langle\tau_{\eta}\right\rangle=18.8$ when weighted by the percentage of occurrences, compared to $\left\langle\tau_{\eta}\right\rangle=38.4$ in regions 1 and 2).

On average, each of the $27^{3}$ Lagrangian trajectories attained the $\ell=1$ state for $0.66 \%$ of its duration. As is shown in the bottom row of Table 3, some $91 \%$ of $\ell=1$ occurrences were solely in regions 1 and 2 . This table shows the eight possible permutations for these two regions. All other cases, which involved regions 3 and 5 in some way, were less frequent than any of the listed eight states. About a third of the occurrences are given by the first row; trajectories already in region 2 that commenced the $\ell=1$ state in region 2 and ended in region 1 . A closely related case where the transition from region 2 to region 1 arose right at the commencement of the $\ell=1$ state (second row), accounted for a further $0.17 / 0.91=19 \%$ of cases. However, the next most common occurrences were associated with a movement counter to that anticipated from the clockwise restricted Euler solution (region 1 to region 2 change in the third row). The fourth row identifies a change from $\ell=0$ to $\ell=1$ while remaining in region 2 . This latter instance (and that where the flow persists in region 1 in the sixth row) highlight the utility of the decomposition into normal and non-normal contributions to the production budget for explaining these dynamics. A transition from region 2 to region 1 (or vice versa) means that $\mathrm{R}_{A}$ has changed sign, demarcating a change in the interplay between enstrophy production and strain production. The cases in the fourth and sixth rows cannot be identified based on sign changes for classic terms. 


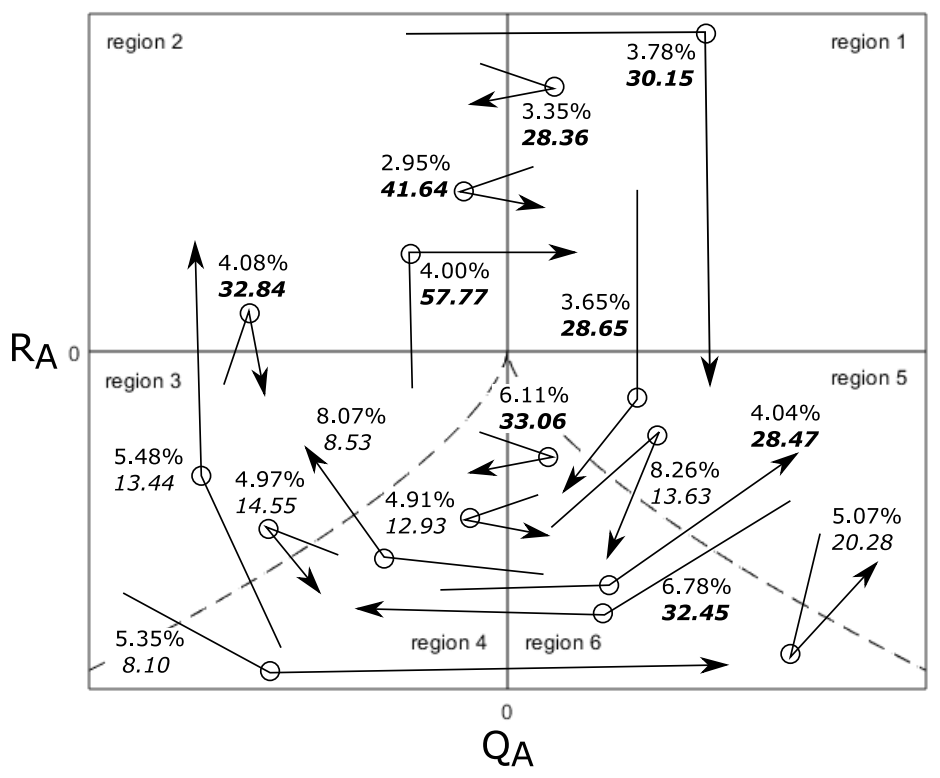

Figure 5: A sketch of the $\mathrm{Q}_{A}-\mathrm{R}_{A}$ diagram with sixteen trajectories superimposed that accounted for over $80 \%$ of recorded trajectories that passed through one of the six regions of $\mathrm{Q}_{A}-\mathrm{R}_{A}$ space. In each case, the circle indicates the region where the trajectory resided, with the region from which it originated $\leq 0.05 \tau_{\eta}$ before the primary region shown by the tail of the arrow, and that $\leq 0.05 \tau$ after it left the primary region shown by the head of the arrow. The label next to each arrow shows the percentage of recorded trajectories associated with this configuration, while the number in italics is the mean time spent in the primary region (in multiples of $\tau_{\eta}$ ). The bold cases are those where this duration exceeded $25 \tau_{\eta}$. 


\subsection{The Lee bound and the sign of $\mathbf{R}_{A}$}

Directly related to the above discussion, there are four possible ways for the third invariant to be zero, two of which are immediately apparent from a conventional analysis: either $-\operatorname{det}\left(S_{A}\right)=\operatorname{tr}\left(\Omega_{A}^{2} S_{A}\right)$, or $\left\|S_{A}\right\| \rightarrow 0$. In addition, however, we may use $(27)$ to note that the other two alternatives are that either the normal strain production and normal enstrophy production are in balance, or $\mathrm{S}_{B} \rightarrow 0$. An inspection of (22) shows that the latter mechanism is the simplest way to obtain $\ell=1$ : A dramatic decline in normal strain production with the non-normal production unaffected and greater in magnitude than interaction production results in $\|\mathrm{N}\|$ remaining approximately constant, with $\left\|S_{B}\right\| \rightarrow 0$ meaning that $\sqrt{2}\left\|S_{A}\right\| \rightarrow\|N\|$ leads to $\ell=1$. In contrast, if the normal enstrophy production and normal strain production terms are in balance, this does not necessarily lead to $\ell=1$ because of a potentially significant contribution to $\sqrt{2}\left\|S_{A}\right\|$ from $\left\|S_{B}\right\|$. It is more straightforward for $S_{B} \rightarrow 0$ than $S_{A} \rightarrow 0$ as the latter requires both the normal and non-normal components of the strain to tend to zero.

This conclusion is supported by the statement in Table 3 that only $0.93 \%$ of the $\ell=1$ states persist for longer than $0.4 \tau_{\eta}$ in the Lagrangian frame, implying that the phenomenon is driven by the behaviour of the local terms rather than the more spatially extensive contributions from the non-local part of the pressure Hessian, which are expected to persist for longer duration scaling more on the duration of a flow structure rather than a fraction of the Kolmogorov time. In addition, because rows four and six of Table 3 show that in $16.5 \%$ of instances the $\ell=1$ state is attained without a change in region, this must mean that the impact of the decline in normal straining on $-\operatorname{det}\left(\mathrm{S}_{B}\right)$ is so much greater than $\operatorname{tr}\left(\Omega_{B}^{2} S_{B}\right)$ that the sign of $R_{A}$ is unaffected, a situation that arises either as a consequence of large normal enstrophy or limited alignment between $\boldsymbol{\omega}_{B}$ and the eigenvectors for $S_{B}$.

It may also be noted that because a decline in $\left\|S_{B}\right\|$ will impact on the interaction production term to a similar degree to the normal enstrophy production (unless there are systematic differences in the strength of alignment between $\boldsymbol{\omega}_{C}$ and the eigenvectors for $S_{B}$ relative to between $\boldsymbol{\omega}_{B}$ and the eigenvectors for $\mathrm{S}_{B}$ ), there is an increased probability that the non-normal production term is the greatest in magnitude of the four production terms. As a consequence, to establish if the mechanism driving these changes is due to the magnitudes of the terms of their alignments, it is important to examine the mutual alignments of $\boldsymbol{\omega}_{B}$ with the eigenvectors for $\mathrm{S}_{B}$, denoted by $\mathbf{e}_{i}^{(B)}$ and ordered with regards to the eigenvalues placed in descending order, and those between $\boldsymbol{\omega}_{C}$ and $\mathbf{e}_{i}^{(B)}$.

\subsection{Alignment structure of the vorticity components and the normal straining}

Denoting the vorticity-strain alignments by $\phi_{j, B}$ where $j \in\{B, C\}$ denotes the relevant vorticity vector, then histograms of the values for $\max \left[\cos \left(\phi_{j, B}\right)\right]$ are given in Fig. 6, with the top row of panels showing results for $\max \left[\cos \left(\phi_{B, B}\right)\right]$ 


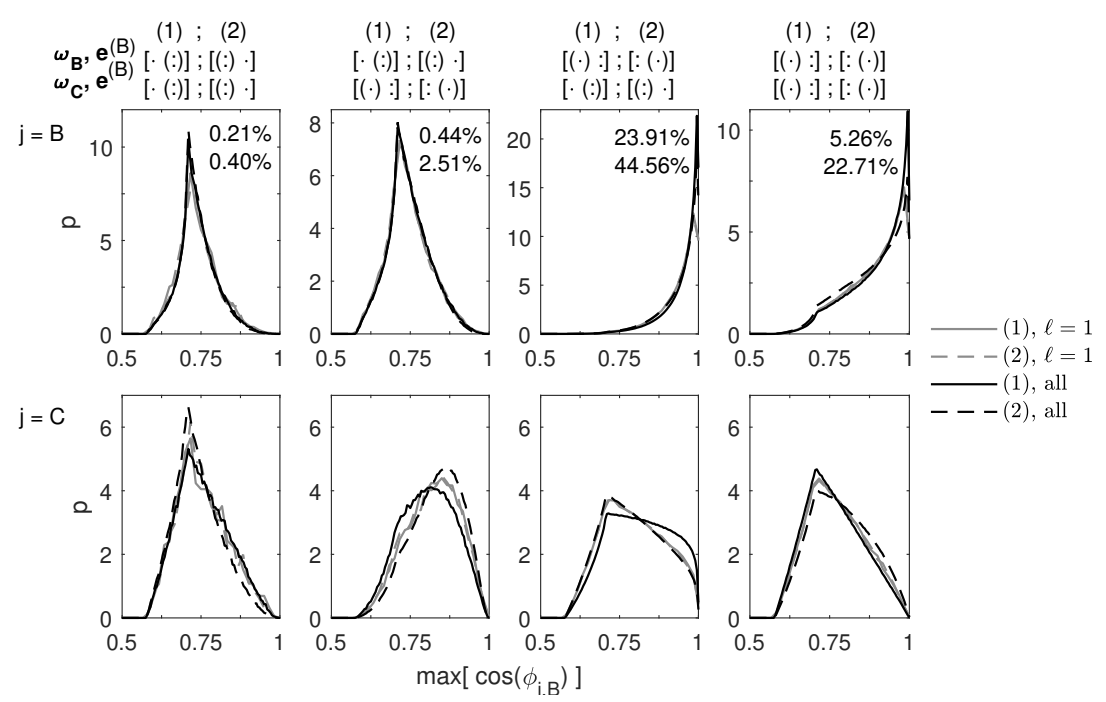

Figure 6: Histograms of the maximum alignment between the vorticity vectors, $\boldsymbol{\omega}_{B}$ (upper row of panels) and $\boldsymbol{\omega}_{C}$ (lower row of panels) and the eigenvectors of the normal strain tensor, $\mathbf{e}_{i}^{(B)}$, for regions 1 and 2 of the $\mathrm{Q}_{A}-\mathrm{R}_{A}$ diagram. The eigenvalues of $S_{B}$ placed in descending rank order are such that either $e_{3}^{(B)}=-2 e_{1}^{(B)}=-2 e_{2}^{(B)}$ (region 1) or $e_{1}^{(B)}=-2 e_{2}^{(B)}=-2 e_{3}^{(B)}$ (region 2), where the former is a rod-like flow structure and the latter is disc-like. Graphically, these are indicated above each panel as [. : $]$ and [: ·. , respectively and results for both regions are shown in each of the eight panels, with the solid lines the results for region 1 and the dashed lines those for region 2 (region numbers are given in brackets in the legend; results at the Lee bound are shown in grey). The four columns of panels segregate which strain eigenvector the vorticity vectors are most aligned with for both $\boldsymbol{\omega}_{B}$ and $\boldsymbol{\omega}_{C}$. This is indicated by the round brackets in the eigenvalue arrangements. Thus, in the second column, and for region 1 , we have $[\cdot(:)]$ for $\max \left[\cos \left(\phi_{B, B}\right)\right]$, the strongest alignment between $\boldsymbol{\omega}_{B}$ and the $\mathbf{e}^{(B)}$, and $[(\cdot):]$ for $\max \left[\cos \left(\phi_{C, B}\right)\right]$. This means that, in this column, results from region 1 have the property that the normal vorticity vector is most aligned with one of the two equal and positive eigenvalues, while the non-normal vorticity vector is most aligned with the only negative eigenvalue. The two percentage values give the relative proportion of all the results shown in each panel for regions 1 (upper value) and 2 (lower value). I.e. over $68 \%$ of the tensors have the alignment properties given by the third column. 
and the bottom row for $\max \left[\cos \left(\phi_{C, B}\right)\right]$. In each panel, results are shown for both regions 1 (solid lines) and region 2 (dashed lines), which necessarily have an opposite configuration for the eigenvalues of $\mathrm{S}_{B}: e_{3}^{(B)}=-2 e_{1}^{(B)}=-2 e_{2}^{(B)}$ (region 1) or $e_{1}^{(B)}=-2 e_{2}^{(B)}=-2 e_{3}^{(B)}$ (region 2 ). These eigenvalues are the real part of those for $B$, which form a conjugate pair and a real value. Hence, we represent the eigenvalues for $S_{B}$ pictorially as [ : :] for region 1 and [: ·] for region 2, respectively. There are then four possibilities for which eigenvector of $S_{B}$ exhibits the greatest alignment: either the eigenvector for the single eigenvalue or for one of the pair of eigenvalues, and for both $\max \left[\cos \left(\phi_{B, B}\right)\right]$ and $\max \left[\cos \left(\phi_{C, B}\right)\right]$. These four states are delimited by the columns in Fig. 6, where the rounded bracket indicates the eigenvalue corresponding to the most aligned eigenvector. The percentages in each upper panel show the relative frequency of each of these possibilities, and it is clear that the most important is the third column where $\max \left[\cos \left(\phi_{B, B}\right)\right]$ occurs for the eigenvector corresponding to the single eigenvalue of $S_{B}$, e.g. [(.) :] and $\max \left[\cos \left(\phi_{C, B}\right)\right]$ for an eigenvector corresponding to one of the paired eigenvalues, e.g. [.(:)]. This makes up $68 \%$ of cases, and is particularly dominant for region 1 ( $23.9 \%$ of the $29.8 \%$ of occurrences in region 1). While the results in columns 1 and 3 have about double the likelihood for region 2 compared to region 1, the occurrences in columns 2 and 4 are four to five times more likely in region 2 highlighting a clear difference in the alignment behaviour for the non-normal component of the vorticity in region 1 . However, from these percentages, it is not clear if this difference also occurs for the $\ell=1$ states. Consequently, the percentage occurrences are further sub-divided in Table 4 and there is a remarkable similarity in the results at the Lee bound irrespective of if the flow is in regions 1 or 2 . Given the typical clock-wise pathway through the $\mathrm{Q}_{A}-\mathrm{R}_{A}$ diagram, on average there is a growth of the state given in the third column at the expense, of those given in the second and fourth columns. Given that the third and fourth columns have the same alignment structure for $\max \left[\cos \left(\phi_{B, B}\right)\right]$ (an alignment with the eigenvector for the single eigenvalue), this transition is primarily about a flip in the alignment structure for $\max \left[\cos \left(\phi_{C, B}\right)\right]$ from one where the alignment is with the eigenvector for the single eigenvalue to one where it is with the pair. Thus, when the normal straining structure is driven towards a topology of forming rod-like structure in region 2 , the probability of both components of vorticity aligning with the extensive eigenvalue is relatively high at $32.4 \%$. When disc-like structures dominate, this probability has halved, and the local and non-local contributions to vorticity are acting in different directions relative to the straining structure. This implies a more unstable behaviour, and provides the physical underpinnings for why compressive flow structures lead to dissipation [Tsinober, 2001].

Given the similarity in the relative frequency of the $\ell=1$ cases between regions 1 and 2, the results in Fig. 6 for the $\ell=1$ cases (grey lines) can be considered directly, without any need to weight the histograms by frequency. It is clear that the distributions for $\max \left[\cos \left(\phi_{B, B}\right)\right]$ have a very similar behaviour in each panel irrespective of if one is considering regions 1 or 2 , or $\ell \in\{0,1\}$. It is also intuitive that there is a tendency to perfect alignment in the third 
Table 4: The percentage of occurrences of different vorticity-strain alignment states for regions 1 and 2 of the $\mathrm{Q}_{A}-\mathrm{R}_{A}$ diagram, adopting the same notation as used in Fig. 6, and isolating the results for $\ell=0$ and $\ell=1$.

$\begin{array}{lrrrr}\max \left[\cos \left(\phi_{B, B}\right)\right] & {[\cdot(:)] \text { or }[(:) \cdot]} & {[\cdot(:)] \text { or }[(:) \cdot]} & {[(\cdot):] \text { or }[:(\cdot)]} & {[(\cdot):] \text { or }[:(\cdot)]} \\ \max \left[\cos \left(\phi_{C, B}\right)\right] & {[\cdot(:)] \text { or }[(:) \cdot]} & {[(\cdot):] \text { or }[:(\cdot)]} & {[\cdot(:)] \text { or }[(:) \cdot]} & {[(\cdot):] \text { or }[:(\cdot)]} \\ \text { Region } 1, \ell=1 & 0.8 \% & 2.4 \% & 70.1 \% & 26.7 \% \\ \text { Region 2, } \ell=1 & 0.8 \% & 2.5 \% & 69.7 \% & 27.1 \% \\ \text { Region } 1, \ell=0 & 0.7 \% & 1.5 \% & 80.4 \% & 17.5 \% \\ \text { Region } 2, \ell=0 & 0.6 \% & 3.6 \% & 63.3 \% & 32.4 \%\end{array}$

and fourth columns where $\max \left[\cos \left(\phi_{B, B}\right)\right]$ involves the single eigenvalue, and $\cos (\pi / 4)=\sqrt{2} / 2$ in the first and second columns where it involves the pair. Based on these results, one might anticipate $\max \left[\cos \left(\phi_{C, B}\right)\right] \rightarrow 1$ in the second and fourth columns where the alignment is with the single eigenvalue, but only in the former case does the mode for the distributions depart from $\sqrt{2} / 2$. Indeed, it the results for region 1 for all data (solid line) in the third column where there is the greatest tendency for $\max \left[\cos \left(\phi_{C, B}\right)\right] \rightarrow 1$, but the alignment here is with one of the pair of eigenvalues. In this panel, the results for $\ell=1 \mathrm{in}$ both regions follow those for region 2. In the other panels in the bottom row, the two histograms for $\ell=1$ are superimposed nearly perfectly and lie in between the results for regions 1 and 2 for all data. This superposition of the alignment histograms for $\ell=1$ in regions 1 and 2 , and the similar relative frequencies in Table 4, mean that one can go further than the results in Fig. 4. The $\ell=1$ state is not only similar across regions 1 and 2 in terms of the sum of the interaction production and non-normal production terms, it is also similar in terms of the alignment structures underpinning these two terms - there is a universal characteristic to the $\ell=1$ state in vortical regions. Furthermore, the broad similarity in the alignment distributions in general means that the differences in the average values for the terms examined in Fig. 3 between the $\ell=0$ and $\ell=1$ states are because of the dramatic decline in $\left\|S_{B}\right\|$.

\subsection{Changes in the contributing terms as $\ell=1$ is attained.}

The results in Fig. 7 show how the attainment of the Lee bound and the associated decline of the magnitude of the local terms impacts on the magnitude of the classical terms: $\left\|\mathrm{S}_{A}\right\|^{2},\left\|\Omega_{A}\right\|^{2},-\operatorname{det}\left(\mathrm{S}_{A}\right)$, and $\operatorname{tr}\left(\Omega_{A}^{2} \mathrm{~S}_{A}\right)$. In every panel in this figure, the very similar nature of the results for "A" and "B" indicate that there is not, on average, any significant, preferred trend for the evolution of these terms over the duration of the $\ell=1$ state as the ratios in the values for the before and after $\ell=1$ states to those during the $\ell=1$ state are similar. Considering regions 3 and 5 first (the bottom group of ten panels), it is clear that the ratios for all the strain-related terms are centred at 1.0; there is no observable impact of the attainment of the bound on the local or traditional 


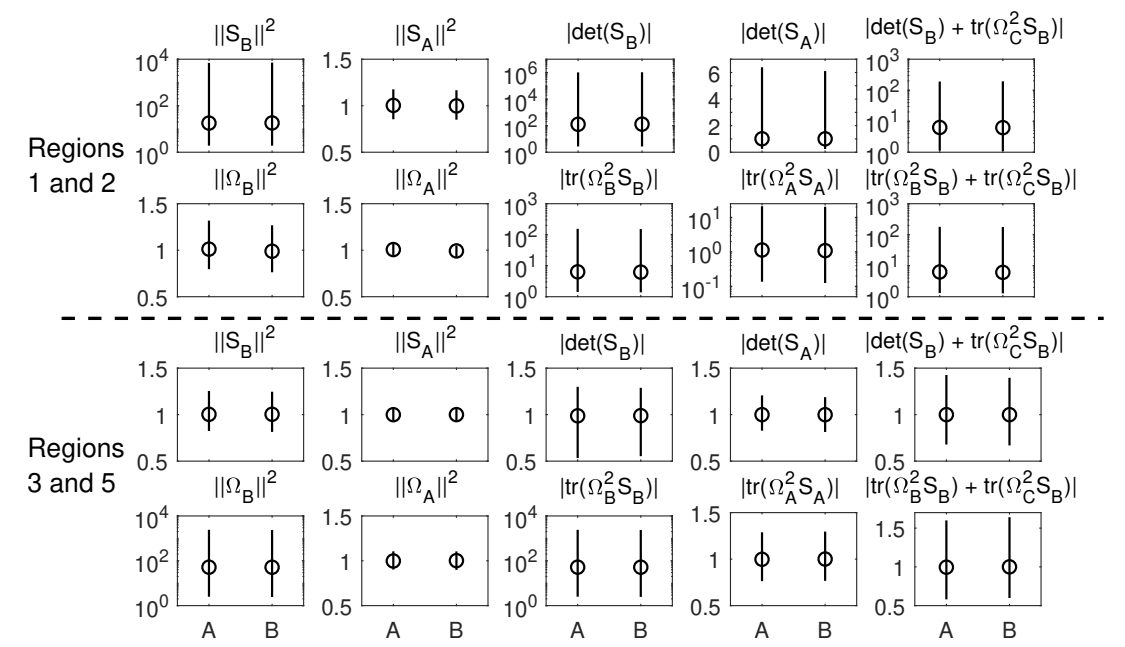

Figure 7: Median values (circles) for the magnitude of the ratio of the term stated above each panel between the flow immediately before the $\ell=1$ state to the mean during that state (A on the abscisssa) and the ratio between the flow immediately after the $\ell=1$ state to the mean during that state (B on the abscissa). The top group of ten panels show results from regions 1 and 2 , while the lower group are for regions 3 and 5 . In each set, the upper row pertains to total strain, strain production and its constituents, while the lower is to enstrophy, enstrophy production and its constituents. The lines in each panel provide the range of values between the $2.5 \%$ and $97.5 \%$ quantiles. 
terms, as anticipated because the bound in these regions is given by the rotation rate. Attainment of the bound results in a typical decline in the magnitudes for both $\left\|\Omega_{B}\right\|^{2}$ and $\operatorname{tr}\left(\Omega_{B}^{2} S_{B}\right)$ by a factor of $\sim 52$. However, this dramatic effect has no noticeable impact on the magnitudes for $\left\|\Omega_{A}\right\|^{2} \operatorname{or} \operatorname{tr}\left(\Omega_{A}^{2} S_{A}\right)$, with values remaining very close to 1.0. Given that there are three terms on the right-hand side of (27), that the change in $\left|\operatorname{tr}\left(\Omega_{B}^{2} \mathrm{~S}_{B}\right)\right|$ is not reflected in $\left|\operatorname{tr}\left(\Omega_{A}^{2} \mathrm{~S}_{A}\right)\right|$ could, in principle, be a consequence of either the interaction production term or the non-normal production term. The right-most panel on the bottom row of Fig. 7 demonstrates that the inclusion of the interaction production term is sufficient to return the ratio to 1.0, i.e. there is no significant average direct effect of non-normal production in this case.

In contrast, the equivalent panel in the results for regions 1 and 2 has a median value of $\sim 6.2$. Hence, in this case, while the ratios for $\left|\operatorname{tr}\left(\Omega_{A}^{2} S_{A}\right)\right|$ are also of the order of 1.0, a strong effect is still present from the combined contribution of the normal enstrophy production and interaction production terms. Thus, in agreement with the results concerning the magnitude of nonnormal production in Fig. 3, the non-normal production is more important for the enstrophy production in the $\ell=1$ states in regions 1 and 2 when the bound is dictated by the strain rate, not the rotation rate. This is despite the weaker decay of the normal enstrophy production in this case by a factor of 6.3 rather than $\sim 52$.

It is the strain-related terms in the top row of Fig. 7 that are affected more directly by the attainment of the bound, with a typical decline in normal total strain by a factor of 18 (about a third of that seen for normal enstrophy in regions 3 and 5), and a decline in normal strain production by a factor of 128 . That this is the greatest relative change for any of the terms follows directly from the fact that this is the only third order constituent determined solely by the term dictating the attainment of the bound. While there are significant departures from a value of 1.0 for the ratios for both $\left|\operatorname{det}\left(\mathrm{S}_{A}\right)\right|$, and $\left|\operatorname{tr}\left(\Omega_{A}^{2} \mathrm{~S}_{A}\right)\right|$ in regions 1 and 2, the median values for the strain production term are once more very close to unity (the two values shown for $\left|\operatorname{det}\left(\mathrm{S}_{A}\right)\right|$ are 1.009 and 1.012; the two for $\left|\operatorname{tr}\left(\Omega_{A}^{2} S_{A}\right)\right|$ are 1.086 and 1.134). Consequently, the key result from Fig. 7 is that the removal of a local contribution to strain production and enstrophy production is hidden in the standard analysis of strain and enstropy production. The median ratios shown for $\left|\operatorname{det}\left(\mathrm{S}_{B}\right)+\operatorname{tr}\left(\Omega_{C}^{2} \mathrm{~S}_{B}\right)\right|$ in regions 1 and 2 shown in the right-most panels are 6.284 and 6.197. This is a significant reduction from 126.7 and 129.7 for $\left|\operatorname{det}\left(S_{B}\right)\right|$, but still significantly different to the median values for $\left|\operatorname{det}\left(S_{A}\right)\right|$. Thus, in this case, both the interaction production and non-normal production terms are important for disguising the absence of a local contribution to the production dynamics, providing support from a Lagrangian perspective to the distribution function for the combination of these terms being the defining feature of the $\ell=1$ states (Fig. 4). 

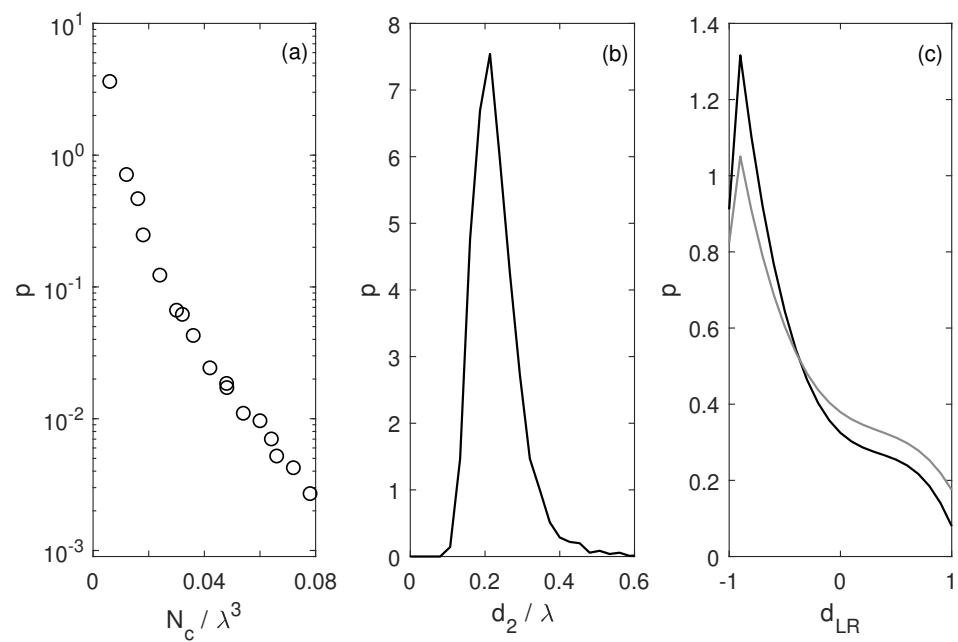

Figure 8: Distribution functions for various measures of the size and shape of regions fulfilling the $\ell=1$ criterion. Panel (a) is the probability density of the number of adjacent VGTs in a cluster, $N_{c}$. Panel (b) gives the intermediate axis, $d_{2}$ for ellipsoids fitted to all the resolved clusters, defined as those consisting of more than than eight VGTs. Panel (c) shows the shape of the resolved clusters applying the normalization of Lund and Rogers [1994] to the principal axes of the fitted ellipsoids, $d_{L R}$ in black. The shape of the flow structures within which these $\ell=1$ clusters are embedded is given by the grey line. This value is dimensionless, while those for $N_{c}$ and $d_{2}$ are non-dimensionalized by the Taylor scale, $\lambda$.

\section{The Size and Configuration of Coherent Re- gions where $\ell=1$}

\subsection{Size and shape of resolved structures with $\ell=1$}

In a Euclidean frame, we extracted the tensors for which $\ell=1$ and then used a 26 connectivity in three dimensions to define, iteratively, groupings of adjacent tensors at the Lee bound. The size of a cluster, $N_{c}$, in terms of the number of adjoining tensors was then determined. Fig. 8a shows the distribution function for the cluster sizes scaled by the cube of the Taylor scale. Defining the minimum resolution of the clustering as a group involving eight tensors (so that the cube root is two tensors), the number density of these resolved clusters was 0.85 $\lambda$. Looking more specifically at the minimum Euclidean distance, $\delta_{E}$ between centroids of these clusters, we obtain the results shown in Fig. 9 which has a median spacing of $0.80 \lambda$, but with sufficient variance and positive skew that values 


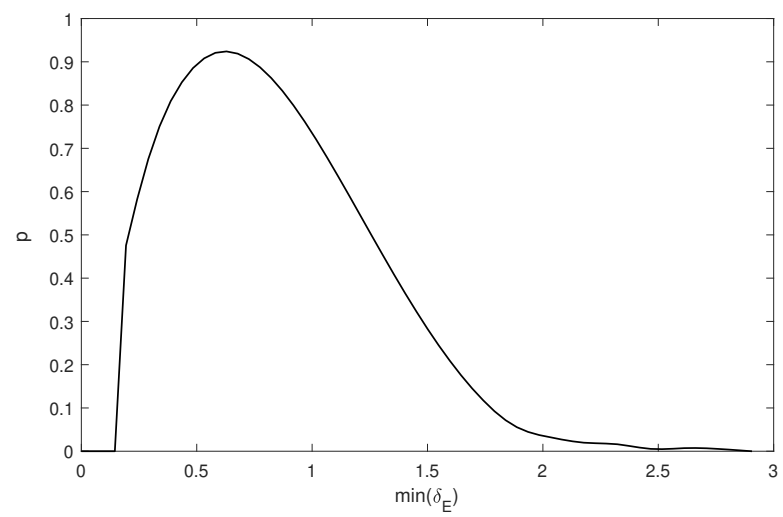

Figure 9: A histogram showing the minimum Euclidean distance between $\ell=1$ clusters, expressed as multiples of the Taylor scale.

of $\delta_{E}=2 \lambda$ are also possible. This presumably reflects, in part, an anisotropy in the shape of the flow structures to which the $\ell=1$ regions are associated. An ellipsoid was fitted to the groups of tensors exceeding the minimum resolution and the three principal axes for the ellipsoid, $d_{i}$ were determined. The mean subtracted values were then derived, $d_{i}^{\prime}=d_{i}-\left\langle d_{i}\right\rangle$, and then the shape of the ellipsoid was determined by using the same normalization that Lund and Rogers [1994] introduced for examining the eigenvalues of the strain rate tensor

$$
d_{L R}=\frac{-3 \sqrt{6} \Pi d_{i}^{\prime}}{\left[\sum\left(d_{i}^{\prime}\right)^{2}\right]^{\frac{3}{2}}}
$$

The distribution for $d_{L R}$ is shown in Fig. $8 \mathrm{c}$ and it is clear that, in contrast to the eigenvalues of the strain rate tensor, which preferentially form disc-like structures [Li and Meneveau, 2007], as may be inferred from the existence of the Vieillefosse tail to the $\mathrm{Q}_{A}-\mathrm{R}_{A}$ diagram [Vieillefosse, 1984], the principal axes of the resolved clusters have a clear tendency to favour tube-like structures, with a median value of $d_{L R}=-0.55$. The elongated nature of these structures helps explain the long positive tail to the distribution in Fig. 9, while a comparison of the black line with the grey line also indicates that this tendency to be elongated is greater on average than that of the flow structures in which such $\ell=1$ events are embedded.

The resolved clusters almost exclusively consisted of $\ell=1$ results for regions 1 and 2, with fewer than $1 \%$ of these clusters having a contribution from regions 3 or 5 . Consequently, occurrences of tensors at the Lee bound in regions 3 and 5 are a much less spatially extensive phenomenon. When they did occur in these resolved clusters, it was a single tensor within the group in $92 \%$ of cases. This difference in spatial extent, combined with the relatively low frequency of La- 
grangian trajectories involving $\ell=1$ tensors in regions 3 and 5 as demonstrated by Table 3 means that the $\ell=1$ occurrences are transient, "one-off" occurrences that arise close to the discriminant function, $\Delta=0$, below which $\left\|\Omega_{B}\right\|=0$ (recall that the bound is dictated by $\Omega_{A}$ for $\mathrm{Q}<0$ ).

In addition to the resolved clusters being overwhelmingly dominated by contributions from regions 1 and 2, it is also of note that the mix of contributions from these two regions was almost equally likely (tensors from region 1 made up $49 \%$ of the tensors in a cluster, with a standard deviation of $7 \%$, a coefficient of skewness of 0.01 and of kurtosis of 3.12). This is in agreement with the results presented earlier that there is a typical behaviour for the flow at the Lee bound in regions 1 and 2, which makes attainment of this state equally likely despite the fact that on average, the flow is in region 2 for 2.4 times as many cases (Fig. 1).

The distribution for the length of the intermediate axis of the fitted ellipsoids is given in Fig. $8 \mathrm{~b}$ and has a mode of $0.23 \lambda$. As $d_{2}$ for the resolved clusters increased, the mean of $49 \%$ occurrences from region 1 stayed the same, meaning that this balance of contributions from region1 and 2 is not a function of size. For example, when only considering cases for which $d_{2}>0.5 \lambda$ (the extreme upper tail as seen in Fig. 8b), the mean was still $49 \%$ with a standard deviation of $3 \%$.

\subsection{The location and orientation of coherent regions where $\ell=1$}

Where extended regions lying at the Lee bound are identified, such occurrrences are completely dominated by region 1 or 2 . Hence, they are innately embedded within coherent flow structures as defined using the simple criterion of $\mathrm{Q}_{A}>0$ [Hunt et al., 1988, Dubief and Delcayre, 2000]. Figure 10 shows the minimum Euclidean distance between the centre of the ellipsoid fitted to the $\ell=1$ regions and that for the centroid of the surrounding coherent structures. This distribution has a mode at approximately one quarter of the radius of the intermediate axis of the larger flow structure. This is true for all cases as well as the most rod-like $\ell=1$ regions where $d_{L R}<-\frac{1}{3}$. Thus, at this distance from the centre, non-local contributions to strain rate and, thus, dissipation are maximal relative to the local dissipation component.

Using the $d_{L R}$ measure for the shape of the ellipsoids, it is clear from the grey lines in Fig. 8c that the coherent structures within which the $\ell=1$ filaments are embedded also tend to have a rod-like shape, although this tendency is weaker than for the $\ell=1$ regions. Denoting the eigenvectors for the ellipsoid for the $\ell=1$ structures as $\mathbf{L}_{j}$ and for the embedded, extended regions that surround them as $\mathbf{R}_{i}$, and with the eigenvectors corresponding to the principal, secondary, tertiary axes ordered from 1 to 3 , the strength of the alignments between these eigenvectors was examined. Results are presented in Fig. 11 as a function of the joint topology of the $\ell=1$ structures, where the value stated first in the panel caption is for the larger scale structure, and that stated second is for the $\ell=1$ structure, where rod and disc are defined by $d_{L R}<-\frac{1}{3}$ 


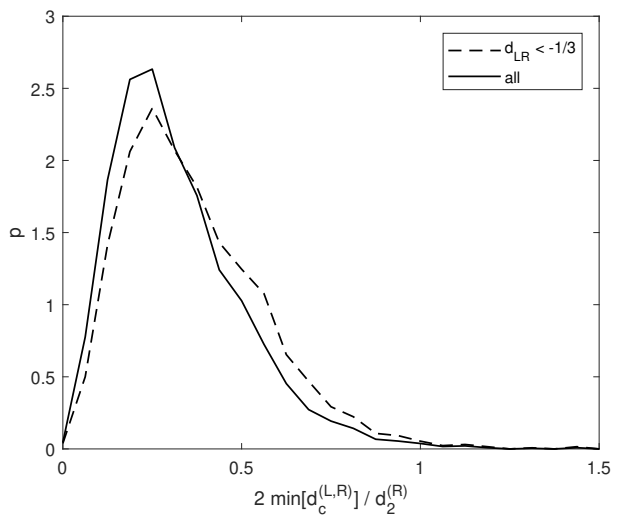

Figure 10: A histogram of the minimum Euclidean distance between the centroid of an $\ell=1$ resolved structure and the centroid of the larger coherent structure within which it is embedded. These distances are non-dimensionlized by the radius of the intermediate axis of the larger structure, $d_{2}^{(R)}$. Results are shown for all $\ell=1$ structures and those which are rod-shaped based on $d_{L R}<-\frac{1}{3}$.

and $d_{L R}>\frac{1}{3}$, respectively. The height of each bar shows the proportion all $\ell=1$ structures where a particular combination of $\mathbf{R}_{i}$ and $\mathbf{L}_{j}$ had the strongest alignment. These are normalized such that the sum over all bars and over all panels is 1.0. That is, the results shown are for $\arg \max \left[\cos \left(\overrightarrow{\mathbf{R}_{i}, \mathbf{L}_{j}}\right)\right]$, where the over-arrow denotes the angle between the underlying terms. Columns with an asterisk are those where the median for $\max \left[\cos \left(\overrightarrow{\mathbf{R}_{i}, \mathbf{L}_{j}}\right)\right]$ was great enough that there was at most $\pm 20^{\circ}$ departure from perfect alignment. Hence, when both ellipsoids are rod-like in shape as shown in panel (a), the most frequently arising dominant alignments are between $\mathbf{R}_{1}$ and $\mathbf{L}_{1}$, followed by $\mathbf{R}_{3}$ and $\mathbf{L}_{3}$. The asterisks also show that the strongest alignments are between $\mathbf{R}_{1}$ and $\mathbf{L}_{1}$ and $\mathbf{R}_{2}$ and $\mathbf{L}_{2}$. In other words, if the coherent structure is rod-like in shape, the rod-like $\ell=1$ structure is aligned with it; both are being extended by the principal axis (most positive eigenvalue). This situation accounts for $61 \%$ of the cases studied as indicated by the percentage in panel (a). The results in panel (b) are qualitatively similar, but have very different topological implications as the disc-like $\ell=1$ region is expanding along its first two axes, but the secondary axis is aligned with a compressing secondary axis for the larger structure. While it is still true in panel (c) that the $\overrightarrow{\mathbf{R}_{1}, \mathbf{L}_{1}}$, and $\overrightarrow{\mathbf{R}_{3}, \mathbf{L}_{3}}$ alignments are most frequent, the typically strongest alignments are for $\overrightarrow{\mathbf{R}_{2}, \mathbf{L}_{1}}$, and $\overrightarrow{\mathbf{R}_{3}, \mathbf{L}_{2}}$, which is a more stable configuration than that in panel (b) as all the associated mean-subtracted eigenvalues have the same sign. This may explain why a rod-like $\ell=1$ filament within a larger disc (21\% of cases) is more frequent than a disc-like $\ell=1$ filament 




Figure 11: The relative frequency of different $\arg \max \left[\cos \left(\overrightarrow{\mathbf{R}_{i}, \mathbf{L}_{j}}\right)\right]$ as a function of the shape of the $\ell=1$ resolved clusters and the coherent structures within which they are embedded. Rods and discs are defined in terms of $d_{L R}<-\frac{1}{3}$ and $d_{L R}>\frac{1}{3}$, respectively. Hence, panel (b) indicates that a $\ell=1$ structure with a disc-like topology is embedded in a $Q_{A}>0$ (i.e. regions 1 or 2 ) structure with a rod-like shape to the ellipsoid fitted to these points. The asterisks in each panel highlight the cases where the median for $\max \left[\cos \left(\overrightarrow{\mathbf{R}}_{i}, \mathbf{L}_{j}\right)\right]$ exceeds 0.9397 (the median angle lies within $\pm 20^{\circ}$ of perfect alignment between the respective eigenvectors. 
within a larger disc-like structure (13\% of cases). The results in panel (d) clearly contrast with the others. The most frequent strongest alignments associated with $\overrightarrow{\mathbf{R}_{2}, \mathbf{L}_{2}}$, and $\overrightarrow{\mathbf{R}_{3}, \mathbf{L}_{3}}$, and the strongest alignments on average occurring for $\overrightarrow{\mathbf{R}_{1}, \mathbf{L}_{2}}$, and $\overrightarrow{\mathbf{R}_{2}, \mathbf{L}_{1}}$. However, again, all these alignments are between meansubtracted eigenvalues of the same sign indicating that this rare configuration ( $5 \%$ of cases) is potentially more stable than that show in panel (b).

\section{The topological nature of the $\ell=1$ tensors}

We identify various types of flow structure by adopting the method of Horiuti [2001]. The starting point for this approach is the vortex identification criterion of Jeong and Hussain [1995], which is pressure-based but may be expressed in terms of the eigenvalues of $\mathrm{H}=\mathrm{S}_{A}^{2}+\Omega_{A}^{2}$. Given a determination of the mutual alignments between the vorticity vector, $\boldsymbol{\omega}_{A}$, and the eigenvectors of $\mathrm{H}, \boldsymbol{e}_{i}^{(H)}$, the most strongly aligned eigenvector is found and its eigenvalue denoted by $e_{z}^{(H)}$. The flow topology is then classified based on the signs of the two remaining eigenvalues:

- $e_{+}^{(H)} \geq e_{-}^{(H)}>0$ - a cylindrical sheet wrapped around a vortex tube (type $C S)$;

- $e_{+}^{(H)} \geq 0 \geq e_{-}^{(H)}$ - a flat sheet where vorticity and strain are comparable (type $F S$ );

- $0>e_{+}^{(H)} \geq e_{-}^{(H)}$ - the core of a vortex tube (type $V T$ ).

Figure 12 shows the nature of the flow topology in each region of the $\mathrm{Q}_{A}-$ $\mathrm{R}_{A}$ diagram for all cases where there was a strong alignment between $\boldsymbol{\omega}_{A}$ and $\boldsymbol{\sigma}_{z}^{(H)}\left[\cos \left(\overrightarrow{\boldsymbol{\omega}_{A}, \boldsymbol{\sigma}_{z}^{(H)}}\right)>0.985\right]$, i.e. $\pm 10^{\circ}$. This equated to $23.7 \%$ of the overall results. In each panel the percentage without brackets is the contribution to $\ell=0$ results in that region, with round brackets is the contribution to $\ell=1$ results in that region, while the value in square brackets is the percentage occupancy of the given region in general. Overall, the total number of $\ell=1$ cases with a strong alignment was $1.6 \%$ of the value for the number of strongly aligned $\ell=0$ occurrences. This indicates a stronger tendency for the $\ell=1$ cases to exhibit such an alignment given the overall relative frequency of $0.66 \%$ quoted at the start of section 4.1 .

It is very clear that the results for regions 1 and 2 indicate the occurrence of vortex tubes, in agreement with the analysis based on $d_{L R}$ in the previous section. The region that exhibits the greatest difference topologically between the $\ell=0$ and $\ell=1$ states is region 5, which yields a flat sheet $78 \%$ of the time and a vortex tube or a curved sheet approximately $11 \%$ of the time in each case for $\ell=0$. In contrast, for $\ell=1$, the curved sheet arises $52 \%$ of the time, and the flat sheet $48 \%$ of the time, which looks much more similar to the $\ell=0$ results for region 6 (59\% : $41 \%$ for curved sheets compared to 



Figure 12: The nature of the flow topology in different regions of the $\mathrm{Q}_{A}-\mathrm{R}_{A}$ diagram for $\ell=0$ cases (dark grey) and $\ell=1$ (light grey). Results are shown for that subset of cases where $\cos \left(\overrightarrow{\boldsymbol{\omega}_{A}, \boldsymbol{e}_{z}^{(H)}}\right)>0.985$. The percentages quoted in each panel are the contribution of that region to the total number of $\ell=0$ cases, the contribution of that region to the $\ell=1$ cases (round brackets), and the overall percentage occupancies for that region, also given in Fig. 1 (square brackets). The labels for three topological states indicate a cylindrical sheet (CS), flat sheet (FS), and a vortex tube (VT). 
flat sheets). Dissipation is known to arise preferentially in the curved sheets that wrap around vortex tubes [Kerr, 1985, Jimenez et al., 1993, Kawahara, 2005, Horiuti et al., 2016] and a high degree of normal straining is particularly prevalent along the Vieillefosse tail between regions 5 and 6 [Keylock, 2018]. Hence, the region $5 \ell=1$ occurrences have a topology that favours dissipation relative to the $\ell=0$ cases in this region.

\section{Conclusion}

A recently introduced approach to studying the dynamics of the velocity gradient tensor (VGT) places a focus on the normal and non-normal components [Keylock, 2018]. As such, it is useful to employ a tight bound on the degree of non-normality [Lee, 1995] to examine those regions in the flow field where non-normality is as large as possible, meaning that viscous effects and/or the anisotropic parts of the pressure Hessian are driving the dynamics. This bound is attained in a trivial fashion where the eigenvalues of the VGT are real because there is no normal contribution to enstrophy, i.e. $\left\|\Omega_{B}\right\|=0$. Regions 3 and 5 lie close to the discriminant function separating real and complex-valued eigenvalues in the $\mathrm{Q}_{A}-\mathrm{R}_{A}$ diagram. Here, the bound is attained in a transitory fashion as seen from how rarely $\ell=1$ occurrences are in these regions (Fig. 1), the absence of any long duration occurrences in these regions (Table 3), and the observation that when such occurrences do arise, they are tyically singular realisations, that do not occur in substantive clusters (discussion in section 5.1).

In contrast, in the enstrophy-dominated regions of the $\mathrm{Q}_{A}-\mathrm{R}_{A}$ diagram, there is a clear structure to the $\ell=1$ occurrences irrespective of the sign of $\mathrm{R}_{A}$. In terms of turbulence production, the PDF of the sum of the interaction and non-normal production terms has a consistent shape that lies closer to that typically arising for region 1, explaining the more efficient generation of $\ell=1$ states in this region (Fig. 4). In addition, the non-normal production term is the greatest in magnitude when $\ell=1$, the opposite of what is generally the case. An examination of the size and shape of the connected regions where $\ell=1$ in regions 1 and 2 indicates that they typically occur in rod-shaped structures, embedded within, and aligned with, rod-shaped coherent flow structures. However, the centre of the $\ell=1$ filaments are typically $\frac{1}{4}$ of the intermediate axis of the larger structure from its centre, and the typical spacing between these filaments is $0.8 \lambda$

Because these filaments typically consist of approximately half their VGTs from regions 1 and 2 , their $\mathrm{Q}_{A}-\mathrm{R}_{A}$ diagram in the enstrophy-dominated regions is symmetric, which is in contrast to what would appear to be the universality of the teardrop shape to the $\mathrm{Q}_{A}-\mathrm{R}_{A}$ diagram for the small-scale statistics of a number of developed or decaying turbulent flows [Laizet et al., 2013, GomesFernandes et al., 2014, Laizet et al., 2015, Zhou et al., 2016, Paul et al., 2017] at high Reynolds number. However, research that uses a modal decomposition such as proper orthogonal decomposition (POD) to define coherent structures from the leading modes has found that the $\mathrm{Q}_{A}-\mathrm{R}_{A}$ for the coherent part also has a high degree of symmetry in the enstrophy-dominated region (e.g. Fig. 6 
of Buxton et al. [2017]). Given that the majority of Lagrangian paths where $\ell=1$ in enstrophy-dominated regions either cross from regions 1 to 2 or vice versa or are initiated just after such a change (Table 3), while they do not typically have their centroid in the centre of a coherent flow structure (Fig.10), the $\ell=1$ regions are, in a sense, the centre of the coherent structure as they represent transitions in the signs of the local enstrophy production and strain production terms. Further work is needed to determine if there is a Reynolds number dependence to the frequency, spatial extent and location of the $\ell=1$ structures relative to the surrounding vortical motion. Such a study would complement recent work by Das and Girimaji [2019] whose investigation into Reynolds number dependencies has suggested a limit of $\operatorname{Re}_{\lambda} \sim 6$ below which the teardrop shape of the $\mathrm{Q}_{A}-\mathrm{R}_{A}$ diagram does not arise for HIT.

The work by Goto and Vassilicos [2009] discussed in the introduction stated that the dissipation coefficient in turbulence is a function of the stagnation point structure, which is in turn a function of the vortical behaviour given that these scale with the Taylor length, $\lambda$, and therefore help structure the location of stagnation points. The typical separation of the centroids of the resolved $\ell=1$ regions is $0.8 \lambda$ (Fig 9) and these filaments within flow structures commonly occur at the interface of regions 1 and 2 where normal enstrophy production and normal strain production are changing sign. That the normal straining is tending to zero in these regions while the overall straining is approximately constant owing to the dominance of the non-local terms in these filaments (Fig. 7), means that the behaviour of the straining field and, thus, dissipation exhibits a subtle behaviour that is not evident using standard analyses. For the production terms, it is the non-normal production that is of greatest importance in such regions even though it is typically the smallest magnitude contribution to the production dynamics as shown in Fig.3.

Given the dominance of non-normal terms in these filaments and the traditional focus in the analysis of the VGT on the invariants of the characteristic equation for the VGT, which are defined in terms of the eigenvalues and, as a consequence, reflect the behaviour of the normal terms [Keylock, 2018], correctly capturing the behaviour of these filaments provides a stringent test for the current drive to develop better models for the dynamics of the velocity gradient tensor [Wilczek and Meneveau, 2014, Johnson and Meneveau, 2016, Das and Girimaji, 2019].

\section{Acknowledgements}

Aspects of this research were supported by Royal Academy of Engineering/Leverhulme Trust Senior Research Fellowship LTSRF1516-12-89. 


\section{References}

W. T. Ashurst, A. R. Kerstein, R. A. Kerr, and C. H. Gibson. Alignment of vorticity and scalar gradient with strain rate in simulated Navier-Stokes turbulence. Phys. Fluids, 30:2343-2353, 1987.

J. G. Ballouz and N. T. Ouellette. Tensor geometry in the turbulent cascade. J. Fluid Mech., 835:1048-1064, 2018. doi: 10.1017/jfm.2017.802.

R. Betchov. An inequality concerning the production of vorticity in isotropic turbulence. J. Fluid Mech., 1:497-504, 1956.

L. Biferale, L. Chevillard, C. Meneveau, and F. Toschi. Multiscale model of gradient evolution in turbulent flows. Phys. Rev. Lett., 98:214501, 2007.

O. R. H. Buxton, M. Breda, and X. Chen. Invariants of the velocity-gradient tensor in a spatially developing inhomogeneous turbulent flow. J. Fluid Mech., $817: 1-20,2017$.

B. J. Cantwell. Exact solution of a restricted Euler equation for the velocity gradient tensor. Phys. Fluids A, 4(4):782-793, 1992.

P. Chakraborty, S. Balachandar, and R. J. Adrian. On the relationships between local vortex identification schemes. J. Fluid Mech., 535:189-214, 2005.

L. Chevillard, C. Meneveau, L. Biferale, and F. Toschi. Modeling the pressure Hessian and viscous Laplacian in turbulence: comparisons with direct numerical simulation and implications on velocity gradient dynamics. Phys. Fluids, 20:101504, 2008.

M. S. Chong, A. E. Perry, and B. J. Cantwell. A general classification of threedimensional flow fields. Phys. Fluids A, 2:765-777, 1990.

R. Das and S. S. Girimaji. On the Reynolds number dependence of velocitygradient structure and dynamics. J. Fluid Mech., 861:163-179, 2019. doi: $10.1017 / \mathrm{jfm} .2018 .924$.

X. Dong, Y. Gao, and C. Liu. New normalized Rortex/vortex identification method. Phys. Fluids, 31:011701, 2019. doi: 10.1063/1.5066016.

Y. Dubief and F. Delcayre. On coherent-vortex identification in turbulence. $J$. Turbul., 1(011), 2000.

P. J. Eberlein. On measures of non-normality for matrices. Amer. Math. Mon., 72:995-996, 1965.

G. E. Elsinga and I. Marusic. Universal aspects of small-scale motions in turbulence. J. Fluid Mech., 662:514-539, 2010. doi: 10.1017/S0022112010003381.

U. Frisch, P. L. Sulem, and M. Nelkin. Simple dynamical model of intermittent fully developed turbulence. J. Fluid Mech., 87:719-736, 1978. 
W. K. George. The decay of homogeneous isotropic turbulence. Phys. Fluids A, 4:1492-1509, 1992. doi: 10.1063/1.858423.

S. S. Girimaji and S. B. Pope. A diffusion model for velocity gradients in turbulence. Phys. Fluids, 2(2):242-256, 1990.

R. Gomes-Fernandes, B. Ganapathisubramani, and J. C. Vassilicos. Evolution of the velocity-gradient tensor in a spatially developing turbulent flow. $J$. Fluid Mech., 756:252-292, 2014.

S. Goto and J. C. Vassilicos. The dissipation rate coefficient of turbulence is not universal and depends on the internal stagnation point structure. Phys. Fluids, 21:035104, 2009.

P. E. Hamlington, J. Schumacher, and W. J. A. Dahm. Direct assessment of vorticity alignment with local and nonlocal strain rates in turbulent flows. Phys. Fluids, 20(111703), 2008. doi: 10.1063/1.3021055.

P. Henrici. Bounds for iterates, inverses, spectral variation and fields of values of non-normal matrices. Numer. Math., 4:24-40, 1962.

K. Horiuti. A classification method for vortex sheet and tube structures in turbulent flows. Phys. Fluids, 13:3756-3774, 2001.

K. Horiuti, S. Yanagihara, and T. Tamaki. Nonequilibrium state in energy spectra and transfer with implications for topological transitions and SGS modeling. Fluid Dyn. Res., 48(021409), 2016.

J. C. R. Hunt, A. A. Wray, and P. Moin. Eddies, stream, and convergence zones in turbulent flows. Technical Report CTR-S88, Center for Turbulence Research, Stanford University, 1988.

J. Jeong and F. Hussain. On the identification of a vortex. J. Fluid Mech., 285: 69-94, 1995.

J. Jimenez, A. A. Wray, P. G. Saffman, and R. S. Rogallo. The structure of intense vorticity in homogeneous isotropic turbulence. J. Fluid Mech., 255: 65-90, 1993.

P. L. Johnson and C. Meneveau. A closure for Lagrangian velocity gradient evolution in turbulence using recent-deformation mapping of initially Gaussian fields. J. Fluid Mech., 804:387-419, 2016.

G. Kawahara. Energy dissipation in spiral vortex layers wrapped around a straight vortex tube. Phys. Fluids, 17(055111), 2005.

R. M. Kerr. Higher-order derivative correlations and the alignment of smallscale structures in isotropic, numerical turbulence. J. Fluid Mech., 153:31-58, 1985 . 
C. J. Keylock. Synthetic velocity gradient tensors and the identification of statistically significant aspects of the structure of turbulence. Phys. Rev. Fluids, 2(004600), 2017.

C. J. Keylock. The Schur decomposition of the velocity gradient tensor for turbulent flows. J. Fluid Mech., 848:876-904, 2018.

A. N. Kolmogorov. The local structure of turbulence in incompressible viscous fluid for very large Reynolds numbers. Dokl. Akad. Nauk. SSSR., 30:299-303, 1941.

A. N. Kolmogorov. A refinement of previous hypotheses concerning the local structure of turbulence in a viscous, incompressible fluid at high Reynolds number. J. Fluid Mech., 13:82-85, 1962.

R. Kress, H. L. De Vies, and R. Wegmann. On nonnormal matrices. Linear Algebra Appl., 8:109-120, 1974.

S. Laizet, J. C. Vassilicos, and C. Cambon. Interscale energy transfer in decaying turbulence and vorticity-strain-rate dynamics in grid-generated turbulence. Fluid Dyn. Res., 45(6):061408, 2013.

S. Laizet, J. Nedić, and C. Vassilicos. Influence of the spatial resolution on finescale features in DNS of turbulence generated by a single square grid. Int. J. Comp. Fluid Dyn., 29(3-5):286-302, 2015.

B. Lashermes, S.G.Roux, P. Abry, and S. Jaffard. Comprehensive multifractal analysis of turbulent velocity using the wavelet leaders. Eur. Phys. J. B, 61: 201-215, 2008. doi: 10.1140/epjb/e2008-00058-4.

S. L. Lee. A practical upper bound for departure from normality. SIAM J. Matrix Analys. Appl., 16:462-468, 1995.

Y. Li and C. Meneveau. Material deformation in a restricted Euler model for turbulent flows: Analytic solution and numerical tests. Phys. Fluids, 19: 015104, 2007. doi: 10.1063/1.2432913.

Y. Li, E. Perlman, M. Wan, Y. Yang, R. Burns, C.Meneveau, S. Chen, A. Szalay, and G. Eyink. A public turbulence database cluster and applications to study Lagrangian evolution of velocity increments in turbulence. J. Turbulence, 9 (31), 2008.

St. Lück, Ch. Renner, J. Peinke, and R. Friedrich. The Markov-Einstein coherence length - a new meaning for the Taylor length in turbulence. Phys. Lett. $A, 359: 335-338,2006$.

T. S. Lund and M. M. Rogers. An improved measure of strain state probability in turbulent flows. Phys. Fluids, 6(5):1838-1847, 1994.

B. Lüthi, M. Holzner, and A. Tsinober. Expanding the Q-R space to three dimensions. J. Fluid Mech., 641:497-507, 2009. 
J. Martin, C. Dopazo, and L. Valiño. Dynamics of velocity gradient invariants in turbulence: restricted Euler and linear diffusion models. Phys. Fluids, 10: 2012-25, 1998.

C. Meneveau. Lagrangian dynamics and models of the velocity gradient tensor in turbulent flows. Ann. Rev. Fluid Mech., 43:219-245, 2011.

C. Meneveau and K.R. Sreenivasan. Simple multifractal cascade model for fully developed turbulence. Phys. Rev. Lett., 59:1424-1427, 1987.

K. Ohkitani. Numerical study of comparison of vorticity and passive vectors in turbulence and inviscid flows. Phys. Rev. E, 65(4):046304, 2002.

K. Ohkitani and S. Kishiba. Nonlocal nature of vortex stretching in an inviscid fluid. Phys. Fluids, 7(2):411-421, 1995.

I. Paul, G. Papadakis, and J. C. Vassilicos. Genesis and evolution of velocity gradients in near-field spatially developing turbulence. J. Fluid Mech., 815: 295-332, 2017. doi: 10.1017/jfm.2017.54.

P. K. Rabey, A. Wynn, and O. R. H. Buxton. The kinematics of the reduced velocity gradient tensor in a fully developed turbulent free shear flow. J. Fluid Mech., 767:627-658, 2015.

I. Schur. Über die charakteristischen Wurzeln einer linearen Substitution mit einer Anwendung auf die Theorie der Integralgleichungen. Math. Ann., 66: 488-510, 1909.

G. I. Taylor. Statistical theory of turbulence. Proc. R. Soc. Lond. A, 151: 421-444, 1935.

G. I. Taylor. The spectrum of turbulence. Proc. R. Soc. Lond. A, 164:476-490, 1938a.

G. I. Taylor. Production and dissipation of vorticity in a turbulent fluid. Proc. R. Soc. Lond. A, 164:15-23, 1938b. doi: 10.1098/rspa.1938.0002.

A. Tsinober. Vortex stretching versus production of strain/dissipation. In J. C. R. Hunt and J. C. Vassilicos, editors, Turbulence Structure and Vortex Dynamics, pages 164-191. Cambridge University Press, 2001.

A. Tsinober. An Informal Conceptual Introduction to Turbulence. Springer, 2009.

P. Vieillefosse. Internal motion of a small element of fluid in an inviscid flow. Physica A, 125:150-162, 1984.

M. Wan, Z. Xiao, C. Meneveau, G. L. Eyink, and S. Chen. Dissipation-energy flux correlations as evidence for the Lagrangian energy cascade in turbulence. Phys. Fluids, 22(6):1-4, 2010. 
M. Wan, S. Chen, G. Eyink, C. Meneveau, E. Perlman, R. Burns, Y. Li, A. Szalay, and S. Hamilton. Johns Hopkins Turbulence Database (JHTDB). http://turbulence.pha.jhu.edu/datasets.aspx, 2016.

M. Wilczek and C. Meneveau. Pressure Hessian and viscous contributions to velocity gradient statistics based on Gaussian random fields. J. Fluid Mech., 756:191-225, 2014.

V. Yakhot. Pressure-velocity correlations and scaling exponents in turbulence. J. Fluid Mech., 495:135-143, 2003.

J. Zhou, R. J. Adrian, S. Balachandar, and T. M. Kendall. Mechanisms for generating coherent packets of hairpin vortices. J. Fluid Mech., 387:353-396, 1999.

Y. Zhou, K. Nagata, Y. Sakai, Y. Ito, and T. Hayase. Spatial evolution of the helical behavior and the $2 / 3$ power-law in single-square-grid-generated turbulence. Fluid Dyn. Res., 48(2):0214042, 2016. 